\title{
Income-related differences in mortality among people with diabetes mellitus
}

\author{
Lorraine L. Lipscombe MD MSc, Peter C. Austin PhD, Douglas G. Manuel MD MSc, \\ Baiju R. Shah MD PhD, Janet E. Hux MD MSc, Gillian L. Booth MD MSc
}

\section{ABSTRACT}

Background: Mortality has declined substantially among people with diabetes mellitus over the last decade. Whether all income groups have benefited equally, however, is unclear. We examined the impact of income on mortality trends among people with diabetes.

Methods: In this population-based, retrospective cohort study, we compared changes in mortality from Apr. 1, 1994, to Mar. 31, 2005, by neighbourhood income strata among people with diabetes aged 30 years or more in the province of Ontario, Canada.

Results: Overall, the annual age- and sex-adjusted mortality declined, from $4.05 \%$ in 1994/95 (95\% confidence interval [Cl] $3.98 \%-4.11 \%$ ) to $2.69 \%$ in $2005 / 06$ (95\% Cl $2.66 \%-$ $2.73 \%)$. The decrease was significantly greater in the highest income group (by 36\%) than in the lowest income group (by $31 \% ; p<0.001$ ). This trend was most pronounced in the younger group (age 30-64 years): the mortality rate ratio widened by more than $40 \%$ between the lowest and highest income groups, from 1.12 to 1.59 among women and from 1.14 to 1.60 among men. Income had a much smaller effect on mortality trends in the older group, whose drug costs are subsidized: the income-related difference rose by only $0.9 \%$ over the study period.

Interpretation: Mortality declined overall among people with diabetes from 1994 to 2005; however, the decrease was substantially greater in the highest income group than in the lowest, particularly among those aged 30-64 years. These findings illustrate the increasing impact of income on the health of people with diabetes even in a publicly funded health care setting. Further studies are needed to explore factors responsible for these income-related differences in mortality.

$\mathrm{T}$ he number of people with diabetes mellitus has increased dramatically over the last 20 years ${ }^{1,2}$ and is estimated to double to about 366 million by $2030 .^{3}$

Diabetes is associated with a 2-fold increase in mortality, with the majority of deaths attributed to cardiovascular causes. ${ }^{4}$ However, survival among people with diabetes has improved substantially over the last decade, ${ }^{1,5,6}$ in part because of better diabetes care and a reduction in cardiovascular events. ${ }^{6}$

Income is a well-known predictor of survival. ${ }^{7,8}$ Even in Canada, where much of health care is universally funded, income-based inequities in health and access to care remain. ${ }^{9-12}$ Although income-related differences in all-cause mortality have decreased since the advent of provincially subsidized health care in Canada, ${ }^{8,9}$ the income gap may be increasing for certain causes of death, including those related to diabetes. ${ }^{8}$ The shift to more complex medical care involving a greater number of drug therapies has resulted in improved diabetes-related outcomes overall. ${ }^{13}$ However, patients in lower-income groups may not have benefited from advances in diabetes care as much as more affluent patients have because of the financial burden of out-of-pocket expenses for such medications and diabetes supplies.

We conducted a population-based study to examine income-related differences in mortality from 1994 to 2005 among people with diabetes.

\section{Methods}

\section{Study design and data sources}

In this population-based, retrospective cohort study, we compared changes in mortality from Apr. 1, 1994, to Mar. 31, 2006, by neighbourhood income strata among people with diabetes aged 30 years or older in Ontario, Canada.

We used Canadian census data and anonymized data from administrative health databases that include records for people covered by the provincial health plan. Health insurance coverage is provided for all Ontario residents; prescription drugs are covered for people aged 65 or more and those receiving social assistance.

We determined the prevalence of diabetes in each study year among people aged 30 years or more using the Ontario Diabetes Database, a registry of Ontario residents with diagnosed diabetes. ${ }^{14}$ With the use of a validated algorithm, people are defined as having diabetes if they have had 1 hospital admission or 2 physician claims bearing a diabetes diagnosis within a 2-year period. ${ }^{14}$ We then grouped people with dia-

From the Institute for Clinical Evaluative Sciences (Lipscombe, Austin, Manuel, Shah, Hux, Booth), Toronto, Ont.; the Department of Medicine (Lipscombe, Shah, Hux, Booth), the Dalla Lana School of Public Health (Austin, Manuel) and the Department of Health Policy, Management and Evauation (Austin, Hux), University of Toronto, Toronto, Ont.; Women's College Hospital (Lipscombe), Toronto, Ont.; Sunnybrook Health Sciences Centre (Shah), Toronto, Ont.; and St. Michael's Hospital (Booth), Toronto, Ont.

CMAJ 2009. DOI:10.1503/cmaj.090495 
betes in each fiscal year into income quintiles by linking their postal codes with Canadian census data, which provide the median household income level of their neighbourhood of residence. ${ }^{9,15}$ We used 1996 census data for the fiscal years 1994 to 1998; 2001 census data for the fiscal years 1999 to 2003; and 2006 census data for the fiscal years 2004 and 2005. People whose postal code could not be linked to a specific neighbourhood income level were assigned to a "missing income" category; mortality data for this group were excluded from the primary analysis but are available in Tables 1, 2 and 3, which appear at the end of this article).

\section{Outcome measures}

We calculated the overall all-cause mortality and the rate for each income quintile in the study population and compared the rates across study years. For numerators, we identified all deaths that occurred in the study population using data from the Registered Persons Database, a provincial registry of demographic and residential information. In this database, deaths are captured from death certificates and supplemented with hospital records of in-hospital deaths.

To adjust for differences in population distribution over time, we performed direct age and sex standardization for mortality (overall and by income quintile) using 1994 population distributions. To explore whether mortality trends differed by age and sex, we calculated unadjusted mortality by age group (those 30-64 years old and those 65 years or more) and sex for each income quintile.

To determine whether trends differed based on diabetes status, we calculated all-cause mortality among people without diabetes in each study year. We determined the denominator for each year using the total Ontario population aged 30 years or more from Canadian census data on July 1 of the previous year, minus the number of people with diabetes that year. We calculated overall rates of death, rates by income quintile and rates by age group and sex within each income quintile. We calculated numerators for mortality by subtracting the number of deaths involving people with diabetes from the corresponding total number of deaths in each year.

\section{Statistical analysis}

We used multivariable Poisson regression analysis to compare mortality by study year, income quintile, age group and sex. We conducted our analyses by fitting a Poisson regression model, relating the variables of interest to the number of deaths, using person-years at risk as an offset. We tested for 2-way and 3-way interactions between age, income and year to determine whether any changes in mortality differed on the basis of income, and whether the effect of income differed between younger people (30-64 years of age) and older people, for whom drug costs are covered. We also compared changes in mortality between populations with and without diabetes to explore whether income-based trends differed by diabetes status. We compared mortality rate ratios between the lowest and highest income quintiles for diabetes patients for each fiscal year. Overall rate ratios were based on age- and sex-adjusted mortality; rate ratios for age and sex subgroups were based on predicted rates from the Poisson regression model.

We conducted a sensitivity analysis that included people who had missing income data, to determine whether differences in mortality among those with missing income data would affect our results. The income quintiles for these individuals were imputed based on age, sex and year. We reanalyzed data with the inclusion of death rates for these people.

\section{Ethics approval}

We obtained ethics approval of our study from the Institutional Review Board at Sunnybrook Health Sciences Centre.

\section{Results}

In 1994/95, a total of 367426 people aged 30 years or more in Ontario had diabetes. This number increased to 843629 by 2005/06, which represents a $130 \%$ relative increase in the number of adults with diabetes over 11 years (Tables 1, 2 and 3 ). During the same period, the population of people in Ontario aged 30 years or more without diabetes increased by 17\%, from 5907012 in 1994/95 to 6888074 in 2005/06 (Tables 4, 5 and 6). (Tables appear at the end of the article.)

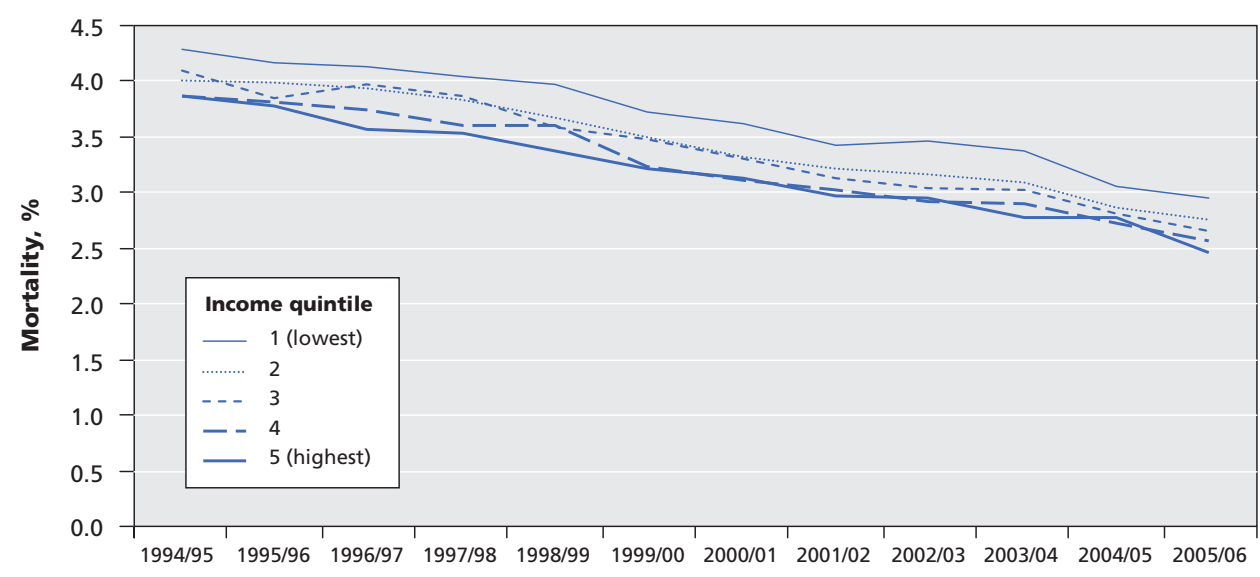

Figure 1: Age- and sex-standardized mortality by income quintile among people in Ontario aged 30 years or more with diabetes mellitus from 1994/95 to 2005/06.

\section{Mortality among people with diabetes}

Rates of death remained significantly higher among men than among women across the years $(4.18 \%$ v. $3.89 \%$ in $1994 / 95$, and $2.86 \%$ v. $2.73 \%$ in $2005 / 06 ; p<$ $0.001)$. Mortality was also significantly higher among people aged 65 years or more than among those aged $30-64(7.14 \%$ v. $1.30 \%$ in $1994 / 95 ; 5.26 \%$ v. $0.79 \%$ in $2005 / 06 ; p<0.001$ ).

In all of the study years, we observed a significant inverse association between income quintile and mortality. Age- and sexstandardized mortality fell signifi- 
cantly, by $33 \%$, across all income quintiles over the study period (Figure 1). Compared with the lowest income quintile, the higher income groups experienced a proportionately greater decline in mortality $(p=0.004$ for interaction between fiscal year and quintile $2 \mathrm{v}$. quintile $1 ; p<0.001$ for interactions with other quintiles $v$. quintile 1). This larger decrease in mortality in the higher income groups was seen mainly among younger individuals (aged 30-64 years) $(p<0.005$ for the interaction between age group, income and year). For instance, among younger women with diabetes, the estimated mortality fell by $45 \%$ in the highest income group, compared with $22 \%$ in the lowest income group (Figure 2, top panel); among the older women with diabetes, income-related differences were much smaller, falling by $22 \%$ in the highest income group and by $21 \%$ in the lowest income group (Figure 2, bottom panel). Trends were similar for men.

\section{Mortality by diabetes status}

The age- and sex-standardized mortality was significantly higher among people with diabetes than among those without the disease across the years (1994/95: $4.04 \%$ v. $0.99 \%, p<0.001$; 2005/06: $2.80 \%$ v. $0.86 \%, p<$ $0.001)$. The decrease in mortality from $1994 / 95$ to $2005 / 06$ was greater among those with diabetes (by 31\%) than among those without it (by 14\%) $(p<0.001)$.

\section{Mortality rate ratio}

The estimated mortality rate ratios between the lowest and highest income quintiles among patients with diabetes across the study years are presented in Figure 3. Although the income-related difference in mortality widened by $8 \%$ overall (from 1.11 to 1.20 ) over the study period, this trend was most pronounced among individuals aged 30-64 years, among whom there was a $40 \%-42 \%$ widening in the rate ratio between the lowest and highest income group. Income had a much smaller effect on mortality trends in the older group, among whom the income-related difference rose by only $0.9 \%$ over the study period.

\section{Sensitivity analysis}

As with our primary analysis, we found a significant interaction between income and mortality across the study years among patients with diabetes when missing income data were included. We also found similar differwere similar among men.) ences in mortality rate ratios between the lowest and highest income quintiles across the study years overall and similar age-related mortality trends (data not shown).

\section{Interpretation}

We found a concerning increase in income-related differences in mortality among people with diabetes in our populationbased study. Overall, mortality decreased by more than $30 \%$ from $1994 / 95$ to $2005 / 06$. However, when we examined trends by income quintile, we found that the improvement in mortality was significantly smaller among people in lowerincome neighbourhoods than among those in wealthier neighbourhoods. This finding was most pronounced among young adults with diabetes, among whom the mortality rate ratio between the lowest and highest income quintile widened by more than $40 \%$. Our findings suggest that improvements in diabetes outcomes may be lagging in the poorer segments of the diabetic population.

There may be several reasons for our findings. First, the
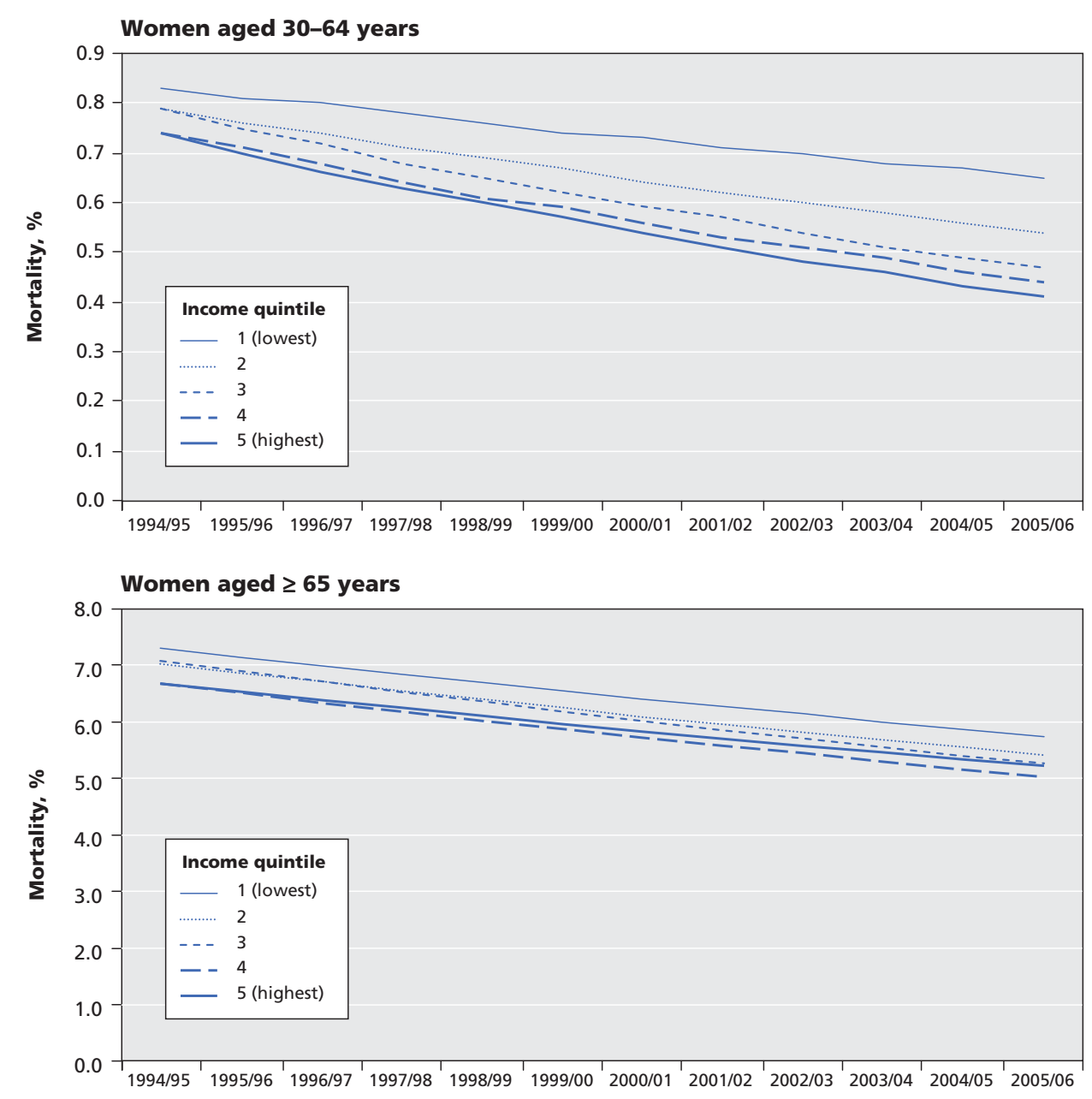

Figure 2: Predicted mortality by income quintile among women with diabetes aged 30-64 years (top panel) and women aged 65 or more (bottom panel) from 1994/95 to 2005/06. (Trends 


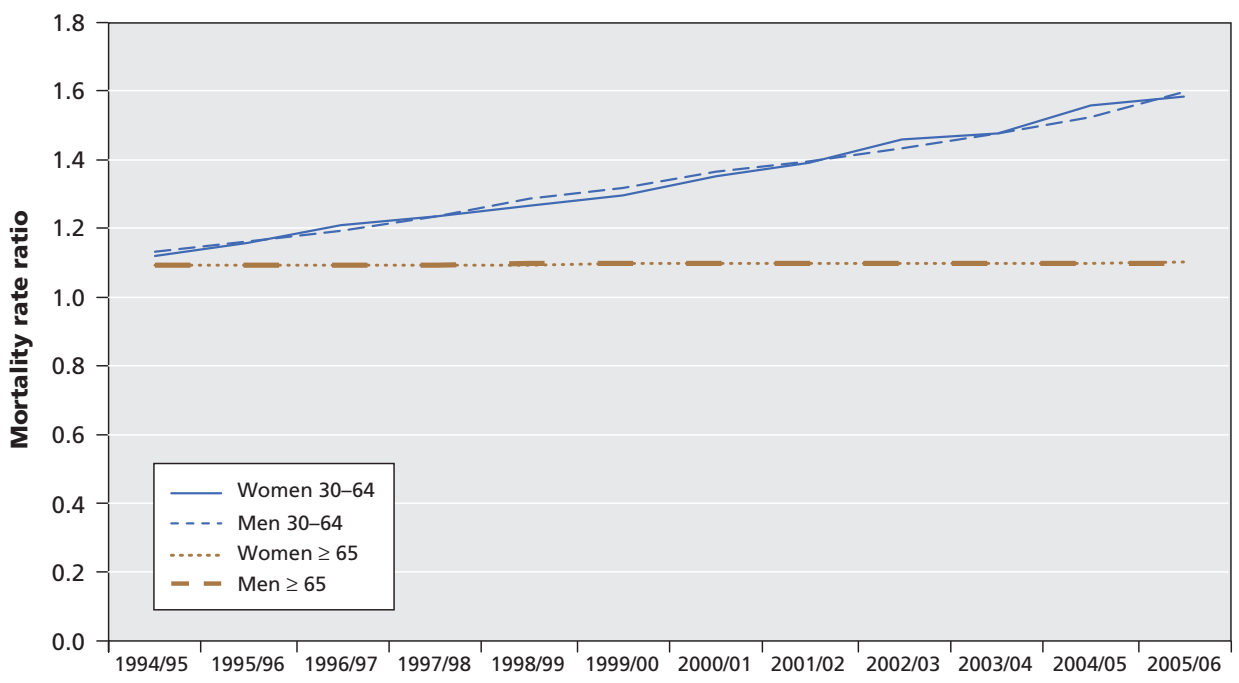

Figure 3: Mortality rate ratios between the lowest and highest income quintile among women and men aged 30 years or more with diabetes from 1994/95 to 2005/06.

adian census data, $28 \%$ of Ontarians were immigrants. ${ }^{25}$ Moreover, an estimated $37 \%$ of people who immigrated during the study period were from South Asia, which represents an increase of more than $50 \%$ since before 1991. ${ }^{25}$ New immigrants often earn less income than longer-term residents, and people of South Asian origin in particular are more susceptible to diabetes and cardiovascular complications. ${ }^{26,27}$

Although increasing socioeconomic inequalities in mortality have been documented in other developed countries, ${ }^{10,11}$ Canada has seen a narrowing of income-related differences in mortality overall since the advent of universal health care. ${ }^{8}$ In con-

complexity and cost of diabetes care has increased markedly over the last decade. ${ }^{13}$ This shift to more intensive treatment has no doubt contributed to an overall improvement in survival, given the proven cardiovascular benefits of multipledrug therapies in patients with type 2 diabetes. ${ }^{16}$ Indeed, the rates of cardiovascular events have declined substantially among patients with diabetes, ${ }^{6}$ and in the Canadian population overall. ${ }^{17}$ Although further studies are needed to determine whether these trends have also differed by income, the increasing cost of medications may now be a greater barrier to effective care for people who cannot afford them. Studies have shown both a rise in the cost of diabetes drugs ${ }^{18}$ and in the number of diabetes patients who cannot afford their medications ${ }^{19,20}$ over the last decade. Indeed, higher out-of-pocket costs for medications have been shown to lower adherence ${ }^{21}$ and increase rates of adverse events. ${ }^{22}$ This is further supported by our finding that income-related differences in mortality were much smaller among people aged 65 years or more, for whom drug costs are subsidized, than among those aged 30-64 years, who do not have universal coverage. Moreover, low health literacy among poorer patients may now have a greater impact on their ability to follow more complex medical regimens. ${ }^{23}$ Lower-income populations are also more susceptible to unhealthy behaviours such as smoking, poor diet and sedentary lifestyle. ${ }^{24}$

Second, the increased gap in mortality observed in our study may have been influenced by differences in rates of screening for diabetes across income groups. Greater screening can have a favourable impact on mortality, since more people with early disease are identified and offered early treatment. Although awareness of diabetes and rates of screening probably increased over the study period, screening rates may have increased more in higher-income groups because of greater education and health advocacy.

Third, the changing ethnic makeup of the Ontario population may have affected our findings. According to 2006 Can- trast, we found a widening gap in income-related differences in mortality among people with diabetes. This is of particular importance given the higher prevalence of diabetes in lowerincome populations. ${ }^{28}$ As diabetes increasingly becomes a disease of lower-income populations, disparities in health outcomes may continue to increase. Our findings may have further implications for Canadians living in rural areas with more limited access to health care. Evidence indicates that, compared with urban residents, people in rural areas have lower income, are more likely to engage in unhealthy behaviours, and have higher rates of diabetes, cardiovascular disease and death. ${ }^{29}$ This trend may also be magnified in nations with greater disparities in access to health care, especially since the biggest increase in diabetes is happening in developing countries. ${ }^{2}$ It is argued that the increased focus on health care as a commercial commodity in many countries has led to greater exclusion of people who cannot pay. ${ }^{30}$ Thus, as rates of diabetes continue to increase globally, this will have major implications for both the health of more vulnerable populations and for resource allocation. Our study highlights the need to address barriers to adequate diabetes care in lowincome populations.

\section{Strengths and limitations}

Strengths of our study include our use of population-based data for a large and diverse jurisdiction and our use of a validated algorithm to identify people with diabetes. However, some limitations merit mention. First, we were unable to differentiate between type 1 and type 2 diabetes. The impact of income on mortality may be different between these groups. Second, we could capture only diagnosed diabetes. Rates of diagnosis may be lower in low-income groups. Third, we did not have data on cause of death and therefore cannot conclude whether the causes of death were similar across income groups. Finally, we did not have income data at the individual level. However, neighbourhood income is a widely used mea- 
sure of socio-economic status that correlates well with individual-level measures and may exert an independent influence on the health of a population. ${ }^{15}$

\section{Conclusion}

Although mortality declined overall among people with diabetes from 1994 to 2005, the decrease was substantially greater in the highest income group than in the lowest. Our findings suggest that wealthier people may have benefited more from advances in diabetes care than poorer people. They also illustrate the increasing impact of income on the health of people with diabetes even in a publicly funded health care setting.

This article has been peer reviewed.

\section{Competing interests: None declared.}

Contributors: All of the authors contributed to the conception and design of the study. Gillian Booth contributed to the study supervision and the acquisition of data. Lorraine Lipscombe, Peter Austin and Douglas Manuel contributed to the analysis and interpretation of data. Lorraine Lipscombe drafted the article, and the other authors critically revised it for important intellectual content. All of the authors gave final approval of the version to be published.

Acknowledgements: The authors thank Ping Li, Institute for Clinical Evaluative Sciences, for assistance with statistical analyses and data presentation; and Pierina Cheung, Women's College Hospital, for assistance with manuscript preparation.

Funding: This study was funded by the National Diabetes Surveillance System fund of the Ontario Ministry of Health and Long-Term Care. The opinions, results and conclusions are those of the authors; no endorsement by the Ministry of Health and Long-Term Care or by the Institute for Clinical Evaluative Sciences is intended or should be inferred. Lorraine Lipscombe is supported by a Canadian Diabetes Association/Canadian Institutes of Health Research (CIHR) Clinician Scientist Award. Peter Austin is supported by a Career Investigator Award from the Heart and Stroke Foundation of Ontario. Douglas Manuel holds a CIHR/Public Health Agency of Canada Chair in Applied Public Health. Baiju Shah receives support from the Canadian Diabetes Association, CIHR and the Banting and Best Diabetes Centre at the University of Toronto. Gillian Booth is supported by a New Investigator Award from the Ontario Women's Health Council and CIHR, and by a Helene and Reuben Dennis Scholar Award from the Banting and Best Diabetes Centre at the University of Toronto.

\section{REFERENCES}

1. Lipscombe LL, Hux JE. Trends in diabetes prevalence, incidence, and mortality in Ontario, Canada 1995-2005: a population-based study. Lancet 2007;369:750-6.

2. Wild S, Roglic G, Green A, et al. Global prevalence of diabetes: estimates for the year 2000 and projections for 2030. Diabetes Care 2004;27:1047-53.

3. World Health Organization. Diabetes programme: facts and figures. Geneva (Switzerland): The Organization; 2008. Available: www.who.int/diabetes/facts/en /index.html (accessed 2009 Oct. 20).

4. Booth GL, Kapral MK, Fung K, et al. Relation between age and cardiovascular disease in men and women with diabetes compared with non-diabetic people: a population-based retrospective cohort study. Lancet 2006;368:29-36.

5. Gregg EW, Gu Q, Cheng YJ, et al. Mortality trends in men and women with diabetes, 1971 to 2000. Ann Intern Med 2007;147:149-55.

6. Booth GL, Kapral MK, Fung K, et al. Recent trends in cardiovascular complications among men and women with and without diabetes. Diabetes Care 2006:29:32-7.

7. Mackenbach JP, Stirbu I, Roskam AJ, et al. Socioeconomic inequalities in health in 22 European countries. N Engl J Med 2008;358:2468-81.

8. Wilkins R, Berthelot JM, Ng E. Trends in mortality by neighbourhood income in urban Canada from 1971 to 1996. Ottawa (ON): Statistics Canada; 2002. Cat no 82-003.

9. Roos NP, Mustard CA. Variation in health and health care use by socioeconomic status in Winnipeg, Canada: Does the system work well? Yes and no. Milbank $Q$ 1997:75:89-111.

10. Pappas G, Queen S, Hadden W, et al. The increasing disparity in mortality between socioeconomic groups in the United States, 1960 and 1986. N Engl J Med 1993;329:103-9.

11. Mackenbach JP, Bos V, Andersen O, et al. Widening socioeconomic inequalities in mortality in six Western European countries. Int J Epidemiol 2003;32:830-7.

12. Alter DA, Naylor CD, Austin P, et al. Effects of socioeconomic status on access to invasive cardiac procedures and on mortality after acute myocardial infarction. N Engl J Med 1999;341:1359-67.

13. Grant RW, Pirraglia PA, Meigs JB, et al. Trends in complexity of diabetes care in the United States from 1991 to 2000. Arch Intern Med 2004;164:1134-9.

14. Hux JE, Ivis F, Flintoft V, et al. Diabetes in Ontario: determination of prevalence and incidence using a validated administrative data algorithm. Diabetes Care 2002;25:512-6.

15. Krieger N. Overcoming the absence of socioeconomic data in medical records: validation and application of a census-based methodology. Am J Public Health 1992; 82:703-10.

16. Gaede $\mathrm{P}$, Lund-Andersen $\mathrm{H}$, Parving $\mathrm{HH}$, et al. Effect of a multifactorial intervention on mortality in type 2 diabetes. $N$ Engl J Med 2008;358:580-91.

17. Tu JV, Nardi L, Fang J, et al. National trends in rates of death and hospital admissions related to acute myocardial infarction, heart failure and stroke, 1994-2004. CMAJ 2009:180:E118-25.

18. Alexander GC, Sehgal NL, Moloney RM, et al. National trends in treatment of type 2 diabetes mellitus, 1994-2007. Arch Intern Med 2008;168:2088-94.

19. Grant RW, McCarthy EP, Singer DE, et al. Frequent outpatient contact and decreasing medication affordability in patients with diabetes from 1997 to 2004 . Diabetes Care 2006;29:1386-8.

20. Kwan J, Razzaq A, Leiter LA, et al. Low socioeconomic status and absence of supplemental health insurance as barriers to diabetes care access and utilization. Canadian Journal of Diabetes 2008;32:174-81.

21. Piette JD, Heisler M, Wagner TH. Problems paying out-of-pocket medication costs among older adults with diabetes. Diabetes Care 2004;27:384-91.

22. Tamblyn R, Laprise R, Hanley JA, et al. Adverse events associated with prescription drug cost-sharing among poor and elderly persons. JAMA 2001;285:421-9.

23. Aikens JE, Piette JD. Diabetic patients' medication underuse, illness outcomes, and beliefs about antihyperglycemic and antihypertensive treatments. Diabetes Care 2009;32:19-24.

24. Pomerleau J, Pederson LL, Ostbye T, et al. Health behaviours and socio-economic status in Ontario, Canada. Eur J Epidemiol 1997;13:613-22.

25. Census of population Ottawa (ON): Statistics Canada; 2006. Cat no 97-562 XCB2006011.

26. Anand SS, Yusuf S, Vuksan V, et al. Differences in risk factors, atherosclerosis, and cardiovascular disease between ethnic groups in Canada: the Study of Health Assessment and Risk in Ethnic groups (SHARE). Lancet 2000;356:279-84.

27. Oza-Frank R, Ali MK, Vaccarino V, et al. Asian-Americans: diabetes prevalence across U.S. and WHO weight classifications. Diabetes Care 2009;32:1644-6.

28. Choi BC, Shi F. Risk factors for diabetes mellitus by age and sex: results of the National Population Health Survey. Diabetologia 2001;44:1221-31.

29. Canadian Institute for Health Information. How healthy are rural Canadians? An assessment of their health status and health determinants: a component of the initiative "Canada's rural communities: understanding rural health and its determinants." Ottawa (ON): Public Health Agency of Canada; 2009.

30. Chan M. Primary health care - now more than ever. Almaty (Kazakhstan): World Health Organization; 2008.

Correspondence to: Dr. Lorraine L. Lipscombe, Women's College Research Institute, 741-790 Bay St., Toronto ON M4N 3M5; lorraine.lipscombe@wchospital.ca

The tables for this article appear on pages E6-E17. 
Table 1: Crude mortality data from $1994 / 95$ to $2005 / 06$ among people with diabetes mellitus aged 30 years or more, by neighbourhood income and age group (part 1 of 2)

\begin{tabular}{|c|c|c|c|c|c|c|c|c|c|c|}
\hline \multirow[b]{2}{*}{ Year } & \multirow[b]{2}{*}{$\begin{array}{l}\text { Income } \\
\text { quintile }\end{array}$} & \multicolumn{3}{|c|}{ All ages ( $\geq 30 \mathrm{yr}$ ) } & \multicolumn{3}{|c|}{ Age 30-64 yr } & \multicolumn{3}{|c|}{ Age $\geq 65 \mathrm{yr}$} \\
\hline & & Deaths & Population & $\begin{array}{c}\text { Mortality, } \\
\%\end{array}$ & Deaths & Population & $\begin{array}{c}\text { Mortality, } \\
\%\end{array}$ & Deaths & Population & $\begin{array}{c}\text { Mortality, } \\
\%\end{array}$ \\
\hline \multirow[t]{7}{*}{$1994 / 95$} & Overall & 14863 & 367426 & 4.05 & 2523 & 195032 & 1.29 & 12340 & 172394 & 7.16 \\
\hline & 1 (lowest) & 3755 & 89104 & 4.21 & 676 & 47058 & 1.44 & 3079 & 42046 & 7.32 \\
\hline & 2 & 3270 & 81113 & 4.03 & 584 & 42608 & 1.37 & 2686 & 38505 & 6.98 \\
\hline & 3 & 3069 & 74078 & 4.14 & 483 & 39100 & 1.24 & 2586 & 34978 & 7.39 \\
\hline & 4 & 2395 & 63447 & 3.77 & 404 & 34531 & 1.17 & 1991 & 28916 & 6.89 \\
\hline & 5 (highest) & 2262 & 57091 & 3.96 & 358 & 30103 & 1.19 & 1904 & 26988 & 7.05 \\
\hline & Missing & 112 & 2593 & 4.32 & 18 & 1632 & 1.10 & 94 & 961 & 9.78 \\
\hline \multirow[t]{7}{*}{$1995 / 96$} & Overall & 15731 & 399574 & 3.94 & 2670 & 212404 & 1.26 & 13061 & 187170 & 6.98 \\
\hline & 1 (lowest) & 3915 & 96215 & 4.07 & 737 & 51179 & 1.44 & 3178 & 45036 & 7.06 \\
\hline & 2 & 3545 & 87795 & 4.04 & 592 & 46071 & 1.28 & 2953 & 41724 & 7.08 \\
\hline & 3 & 3129 & 80423 & 3.89 & 489 & 42398 & 1.15 & 2640 & 38025 & 6.94 \\
\hline & 4 & 2606 & 69826 & 3.73 & 424 & 38103 & 1.11 & 2182 & 31723 & 6.88 \\
\hline & 5 (highest) & 2423 & 62549 & 3.87 & 402 & 32888 & 1.22 & 2021 & 29661 & 6.81 \\
\hline & Missing & 113 & 2766 & 4.09 & 26 & 1765 & 1.47 & 87 & 1001 & 8.69 \\
\hline \multirow[t]{7}{*}{ 1996/97 } & Overall & 16878 & 431524 & 3.91 & 2603 & 229438 & 1.13 & 14275 & 202086 & 7.06 \\
\hline & 1 (lowest) & 4152 & 102911 & 4.03 & 700 & 55240 & 1.27 & 3452 & 47671 & 7.24 \\
\hline & 2 & 3771 & 94124 & 4.01 & 617 & 49284 & 1.25 & 3154 & 44840 & 7.03 \\
\hline & 3 & 3530 & 87188 & 4.05 & 519 & 45877 & 1.13 & 3011 & 41311 & 7.29 \\
\hline & 4 & 2800 & 76042 & 3.68 & 423 & 41310 & 1.02 & 2377 & 34732 & 6.84 \\
\hline & 5 (highest) & 2522 & 68161 & 3.70 & 326 & 35697 & 0.91 & 2196 & 32464 & 6.76 \\
\hline & Missing & 103 & 3098 & 3.32 & 18 & 2030 & 0.89 & 85 & 1068 & 7.96 \\
\hline \multirow[t]{7}{*}{$1997 / 98$} & Overall & 17784 & 467194 & 3.81 & 2754 & 249399 & 1.10 & 15030 & 217795 & 6.90 \\
\hline & 1 (lowest) & 4289 & 109951 & 3.90 & 769 & 59796 & 1.29 & 3520 & 50155 & 7.02 \\
\hline & 2 & 3963 & 101403 & 3.91 & 594 & 53258 & 1.12 & 3369 & 48145 & 7.00 \\
\hline & 3 & 3745 & 94534 & 3.96 & 542 & 49716 & 1.09 & 3203 & 44818 & 7.15 \\
\hline & 4 & 2970 & 83474 & 3.56 & 439 & 45336 & 0.97 & 2531 & 38138 & 6.64 \\
\hline & 5 (highest) & 2722 & 74471 & 3.66 & 393 & 39066 & 1.01 & 2329 & 35405 & 6.58 \\
\hline & Missing & 95 & 3361 & 2.83 & 17 & 2227 & 0.76 & 78 & 1134 & 6.88 \\
\hline \multirow[t]{7}{*}{$1998 / 99$} & Overall & 18472 & 502725 & 3.67 & 2857 & 269170 & 1.06 & 15615 & 233555 & 6.69 \\
\hline & 1 (lowest) & 4499 & 117143 & 3.84 & 783 & 64066 & 1.22 & 3716 & 53077 & 7.00 \\
\hline & 2 & 4063 & 108524 & 3.74 & 630 & 57142 & 1.10 & 3433 & 51382 & 6.68 \\
\hline & 3 & 3727 & 101710 & 3.66 & 551 & 53689 & 1.03 & 3176 & 48021 & 6.61 \\
\hline & 4 & 3224 & 90989 & 3.54 & 473 & 49655 & 0.95 & 2751 & 41334 & 6.66 \\
\hline & 5 (highest) & 2853 & 80666 & 3.54 & 401 & 42225 & 0.95 & 2452 & 38441 & 6.38 \\
\hline & Missing & 106 & 3693 & 2.87 & 19 & 2393 & 0.79 & 87 & 1300 & 6.69 \\
\hline \multirow[t]{7}{*}{$1999 / 00$} & Overall & 18761 & 541224 & 3.47 & 2993 & 291121 & 1.03 & 15768 & 250103 & 6.30 \\
\hline & 1 (lowest) & 4786 & 130222 & 3.68 & 802 & 70766 & 1.13 & 3984 & 59456 & 6.70 \\
\hline & 2 & 4437 & 122253 & 3.63 & 663 & 63655 & 1.04 & 3774 & 58598 & 6.44 \\
\hline & 3 & 3735 & 106916 & 3.49 & 603 & 57076 & 1.06 & 3132 & 49840 & 6.28 \\
\hline & 4 & 2908 & 93101 & 3.12 & 513 & 51531 & 1.00 & 2395 & 41570 & 5.76 \\
\hline & 5 (highest) & 2817 & 84828 & 3.32 & 391 & 45372 & 0.86 & 2426 & 39456 & 6.15 \\
\hline & Missing & 78 & 3904 & 2.00 & 21 & 2721 & 0.77 & 57 & 1183 & 4.82 \\
\hline
\end{tabular}


Table 1: Crude mortality data from $1994 / 95$ to $2005 / 06$ among people with diabetes mellitus aged 30 years or more, by neighbourhood income and age group (part 2 of 2)

\begin{tabular}{|c|c|c|c|c|c|c|c|c|c|c|}
\hline \multirow[b]{2}{*}{ Year } & \multirow[b]{2}{*}{$\begin{array}{l}\text { Income } \\
\text { quintile }\end{array}$} & \multicolumn{3}{|c|}{ All ages ( $\geq 30 \mathrm{yr}$ ) } & \multicolumn{3}{|c|}{ Age 30-64 yr } & \multicolumn{3}{|c|}{ Age $\geq 65 \mathrm{yr}$} \\
\hline & & Deaths & Population & $\begin{array}{c}\text { Mortality, } \\
\%\end{array}$ & Deaths & Population & $\begin{array}{c}\text { Mortality, } \\
\%\end{array}$ & Deaths & Population & $\begin{array}{c}\text { Mortality, } \\
\%\end{array}$ \\
\hline \multirow[t]{7}{*}{$2000 / 01$} & Overall & 19541 & 582113 & 3.36 & 3036 & 314305 & 0.97 & 16505 & 267808 & 6.16 \\
\hline & 1 (lowest) & 4983 & 138383 & 3.60 & 884 & 75544 & 1.17 & 4099 & 62839 & 6.52 \\
\hline & 2 & 4541 & 130713 & 3.47 & 668 & 68197 & 0.98 & 3873 & 62516 & 6.20 \\
\hline & 3 & 3856 & 115519 & 3.34 & 559 & 62084 & 0.90 & 3297 & 53435 & 6.17 \\
\hline & 4 & 3082 & 101674 & 3.03 & 517 & 56431 & 0.92 & 2565 & 45243 & 5.67 \\
\hline & 5 (highest) & 3008 & 91741 & 3.28 & 389 & 49196 & 0.79 & 2619 & 42545 & 6.16 \\
\hline & Missing & 71 & 4083 & 1.74 & 19 & 2853 & 0.67 & 52 & 1230 & 4.23 \\
\hline \multirow[t]{7}{*}{$2001 / 02$} & Overall & 20275 & 630293 & 3.22 & 3193 & 342576 & 0.93 & 17082 & 287717 & 5.94 \\
\hline & 1 (lowest) & 5059 & 147692 & 3.43 & 864 & 80948 & 1.07 & 4195 & 66744 & 6.29 \\
\hline & 2 & 4752 & 140853 & 3.37 & 720 & 73912 & 0.97 & 4032 & 66941 & 6.02 \\
\hline & 3 & 3957 & 125240 & 3.16 & 594 & 67952 & 0.87 & 3363 & 57288 & 5.87 \\
\hline & 4 & 3299 & 111967 & 2.95 & 552 & 62457 & 0.88 & 2747 & 49510 & 5.55 \\
\hline & 5 (highest) & 3109 & 100223 & 3.10 & 429 & 54320 & 0.79 & 2680 & 45903 & 5.84 \\
\hline & Missing & 99 & 4318 & 2.29 & 34 & 2987 & 1.14 & 65 & 1331 & 4.88 \\
\hline \multirow[t]{7}{*}{$2002 / 03$} & Overall & 21619 & 680277 & 3.18 & 3421 & 372005 & 0.92 & 18198 & 308272 & 5.90 \\
\hline & 1 (lowest) & 5442 & 156570 & 3.48 & 982 & 86092 & 1.14 & 4460 & 70478 & 6.33 \\
\hline & 2 & 5028 & 150889 & 3.33 & 762 & 79739 & 0.96 & 4266 & 71150 & 6.00 \\
\hline & 3 & 4195 & 135856 & 3.09 & 613 & 74142 & 0.83 & 3582 & 61714 & 5.80 \\
\hline & 4 & 3503 & 123097 & 2.85 & 565 & 69260 & 0.82 & 2938 & 53837 & 5.46 \\
\hline & 5 (highest) & 3367 & 109251 & 3.08 & 479 & 59549 & 0.80 & 2888 & 49702 & 5.81 \\
\hline & Missing & 84 & 4614 & 1.82 & 20 & 3223 & 0.62 & 64 & 1391 & 4.60 \\
\hline \multirow[t]{7}{*}{$2003 / 04$} & Overall & 22678 & 728077 & 3.11 & 3596 & 399999 & 0.90 & 19082 & 328078 & 5.82 \\
\hline & 1 (lowest) & 5527 & 164018 & 3.37 & 995 & 90499 & 1.10 & 4532 & 73519 & 6.16 \\
\hline & 2 & 5252 & 159989 & 3.28 & 818 & 84850 & 0.96 & 4434 & 75139 & 5.90 \\
\hline & 3 & 4524 & 146388 & 3.09 & 692 & 80380 & 0.86 & 3832 & 66008 & 5.81 \\
\hline & 4 & 3863 & 134592 & 2.87 & 601 & 75949 & 0.79 & 3262 & 58643 & 5.56 \\
\hline & 5 (highest) & 3409 & 118016 & 2.89 & 463 & 64812 & 0.71 & 2946 & 53204 & 5.54 \\
\hline & Missing & 103 & 5074 & 2.03 & 27 & 3509 & 0.77 & 76 & 1565 & 4.86 \\
\hline \multirow[t]{7}{*}{$2004 / 05$} & Overall & 22977 & 782333 & 2.94 & 3551 & 431067 & 0.82 & 19426 & 351266 & 5.53 \\
\hline & 1 (lowest) & 5742 & 187281 & 3.07 & 1039 & 104915 & 0.99 & 4703 & 82366 & 5.71 \\
\hline & 2 & 5103 & 171899 & 2.97 & 770 & 93350 & 0.82 & 4333 & 78549 & 5.52 \\
\hline & 3 & 4346 & 151924 & 2.86 & 653 & 83799 & 0.78 & 3693 & 68125 & 5.42 \\
\hline & 4 & 3954 & 141582 & 2.79 & 573 & 78217 & 0.73 & 3381 & 63365 & 5.34 \\
\hline & 5 (highest) & 3736 & 125502 & 2.98 & 492 & 68117 & 0.72 & 3244 & 57385 & 5.65 \\
\hline & Missing & 96 & 4145 & 2.32 & 24 & 2669 & 0.90 & 72 & 1476 & 4.88 \\
\hline \multirow[t]{7}{*}{$2005 / 06$} & Overall & 23552 & 843629 & 2.79 & 3649 & 465381 & 0.78 & 19903 & 378248 & 5.26 \\
\hline & 1 (lowest) & 5930 & 198473 & 2.99 & 1062 & 111176 & 0.96 & 4868 & 87297 & 5.58 \\
\hline & 2 & 5291 & 184737 & 2.86 & 827 & 100490 & 0.82 & 4464 & 84247 & 5.30 \\
\hline & 3 & 4547 & 165830 & 2.74 & 678 & 91656 & 0.74 & 3869 & 74174 & 5.22 \\
\hline & 4 & 4069 & 154211 & 2.64 & 598 & 85348 & 0.70 & 3471 & 68863 & 5.04 \\
\hline & 5 (highest) & 3620 & 135723 & 2.67 & 471 & 73719 & 0.64 & 3149 & 62004 & 5.08 \\
\hline & Missing & 95 & 4655 & 2.04 & 13 & 2992 & 0.43 & 82 & 1663 & 4.93 \\
\hline
\end{tabular}


Table 2: Crude mortality data from $1994 / 95$ to $2005 / 06$ among women with diabetes mellitus aged 30 years or more, by neighbourhood income and age group (part 1 of 2 )

\begin{tabular}{|c|c|c|c|c|c|c|c|c|c|c|}
\hline \multirow[b]{2}{*}{ Year } & \multirow[b]{2}{*}{$\begin{array}{l}\text { Income } \\
\text { quintile }\end{array}$} & \multicolumn{3}{|c|}{ All ages ( $\geq 30 \mathrm{yr}$ ) } & \multicolumn{3}{|c|}{ Age $30-64$ yr } & \multicolumn{3}{|c|}{ Age $\geq 65 \mathrm{yr}$} \\
\hline & & Deaths & Population & $\begin{array}{c}\text { Mortality, } \\
\%\end{array}$ & Deaths & Population & $\begin{array}{c}\text { Mortality, } \\
\%\end{array}$ & Deaths & Population & $\begin{array}{c}\text { Mortality, } \\
\%\end{array}$ \\
\hline \multirow[t]{7}{*}{$1994 / 95$} & Overall & 6884 & 176569 & 3.90 & 903 & 86355 & 1.05 & 5981 & 90214 & 6.63 \\
\hline & 1 (lowest) & 1835 & 46474 & 3.95 & 267 & 22587 & 1.18 & 1568 & 23887 & 6.56 \\
\hline & 2 & 1512 & 39705 & 3.81 & 209 & 19232 & 1.09 & 1303 & 20473 & 6.36 \\
\hline & 3 & 1427 & 35171 & 4.06 & 177 & 17145 & 1.03 & 1250 & 18026 & 6.93 \\
\hline & 4 & 1067 & 28901 & 3.69 & 140 & 14471 & 0.97 & 927 & 14430 & 6.42 \\
\hline & 5 (highest) & 980 & 25027 & 3.92 & 107 & 12151 & 0.88 & 873 & 12876 & 6.78 \\
\hline & Missing & 63 & 1291 & 4.88 & 3 & 769 & 0.39 & 60 & 522 & 11.49 \\
\hline \multirow[t]{7}{*}{ 1995/96 } & Overall & 7197 & 191782 & 3.75 & 976 & 94188 & 1.04 & 6221 & 97594 & 6.37 \\
\hline & 1 (lowest) & 1874 & 50016 & 3.75 & 302 & 24578 & 1.23 & 1572 & 25438 & 6.18 \\
\hline & 2 & 1611 & 43000 & 3.75 & 213 & 20846 & 1.02 & 1398 & 22154 & 6.31 \\
\hline & 3 & 1385 & 38232 & 3.62 & 157 & 18667 & 0.84 & 1228 & 19565 & 6.28 \\
\hline & 4 & 1163 & 31787 & 3.66 & 141 & 16009 & 0.88 & 1022 & 15778 & 6.48 \\
\hline & 5 (highest) & 1106 & 27358 & 4.04 & 154 & 13258 & 1.16 & 952 & 14100 & 6.75 \\
\hline & Missing & 58 & 1389 & 4.18 & 9 & 830 & 1.08 & 49 & 559 & 8.77 \\
\hline \multirow[t]{7}{*}{$1996 / 97$} & Overall & 7897 & 207392 & 3.81 & 940 & 102067 & 0.92 & 6957 & 105325 & 6.61 \\
\hline & 1 (lowest) & 2025 & 53653 & 3.77 & 259 & 26606 & 0.97 & 1766 & 27047 & 6.53 \\
\hline & 2 & 1744 & 46220 & 3.77 & 240 & 22435 & 1.07 & 1504 & 23785 & 6.32 \\
\hline & 3 & 1652 & 41567 & 3.97 & 184 & 20289 & 0.91 & 1468 & 21278 & 6.90 \\
\hline & 4 & 1291 & 34641 & 3.73 & 136 & 17414 & 0.78 & 1155 & 17227 & 6.70 \\
\hline & 5 (highest) & 1135 & 29786 & 3.81 & 115 & 14378 & 0.80 & 1020 & 15408 & 6.62 \\
\hline & Missing & 50 & 1525 & 3.28 & 6 & 945 & 0.63 & 44 & 580 & 7.59 \\
\hline \multirow[t]{7}{*}{$1997 / 98$} & Overall & 8297 & 224770 & 3.69 & 951 & 111514 & 0.85 & 7346 & 113256 & 6.49 \\
\hline & 1 (lowest) & 2047 & 57129 & 3.58 & 271 & 28855 & 0.94 & 1776 & 28274 & 6.28 \\
\hline & 2 & 1848 & 49852 & 3.71 & 204 & 24299 & 0.84 & 1644 & 25553 & 6.43 \\
\hline & 3 & 1791 & 45284 & 3.96 & 197 & 22101 & 0.89 & 1594 & 23183 & 6.88 \\
\hline & 4 & 1345 & 38193 & 3.52 & 135 & 19331 & 0.70 & 1210 & 18862 & 6.42 \\
\hline & 5 (highest) & 1218 & 32605 & 3.74 & 137 & 15844 & 0.86 & 1081 & 16761 & 6.45 \\
\hline & Missing & 48 & 1707 & 2.81 & 7 & 1084 & 0.65 & 41 & 623 & 6.58 \\
\hline \multirow[t]{7}{*}{$1998 / 99$} & Overall & 8614 & 241817 & 3.56 & 1042 & 120768 & 0.86 & 7572 & 121049 & 6.26 \\
\hline & 1 (lowest) & 2234 & 60772 & 3.68 & 303 & 30846 & 0.98 & 1931 & 29926 & 6.45 \\
\hline & 2 & 1882 & 53564 & 3.51 & 227 & 26230 & 0.87 & 1655 & 27334 & 6.05 \\
\hline & 3 & 1732 & 48659 & 3.56 & 200 & 23999 & 0.83 & 1532 & 24660 & 6.21 \\
\hline & 4 & 1442 & 41629 & 3.46 & 177 & 21286 & 0.83 & 1265 & 20343 & 6.22 \\
\hline & 5 (highest) & 1260 & 35335 & 3.57 & 125 & 17262 & 0.72 & 1135 & 18073 & 6.28 \\
\hline & Missing & 64 & 1858 & 3.44 & 10 & 1145 & 0.87 & 54 & 713 & 7.57 \\
\hline \multirow[t]{7}{*}{$1999 / 00$} & Overall & 8747 & 260244 & 3.36 & 1096 & 131012 & 0.84 & 7651 & 129232 & 5.92 \\
\hline & 1 (lowest) & 2341 & 67688 & 3.46 & 325 & 34250 & 0.95 & 2016 & 33438 & 6.03 \\
\hline & 2 & 2092 & 60196 & 3.48 & 253 & 29336 & 0.86 & 1839 & 30860 & 5.96 \\
\hline & 3 & 1691 & 50487 & 3.35 & 212 & 25276 & 0.84 & 1479 & 25211 & 5.87 \\
\hline & 4 & 1295 & 42616 & 3.04 & 178 & 22161 & 0.80 & 1117 & 20455 & 5.46 \\
\hline & 5 (highest) & 1292 & 37253 & 3.47 & 118 & 18596 & 0.63 & 1174 & 18657 & 6.29 \\
\hline & Missing & 36 & 2004 & 1.80 & 10 & 1393 & 0.72 & 26 & 611 & 4.26 \\
\hline
\end{tabular}


Table 2: Crude mortality data from $1994 / 95$ to 2005/06 among women with diabetes mellitus aged 30 years or more, by neighbourhood income and age group (part 2 of 2)

\begin{tabular}{|c|c|c|c|c|c|c|c|c|c|c|}
\hline \multirow[b]{2}{*}{ Year } & \multirow[b]{2}{*}{$\begin{array}{l}\text { Income } \\
\text { quintile }\end{array}$} & \multicolumn{3}{|c|}{ All ages ( $\geq 30 \mathrm{yr}$ ) } & \multicolumn{3}{|c|}{ Age $30-64$ yr } & \multicolumn{3}{|c|}{ Age $\geq 65 y r$} \\
\hline & & Deaths & Population & $\begin{array}{c}\text { Mortality, } \\
\%\end{array}$ & Deaths & Population & $\begin{array}{c}\text { Mortality, } \\
\%\end{array}$ & Deaths & Population & $\begin{array}{c}\text { Mortality, } \\
\%\end{array}$ \\
\hline \multirow[t]{7}{*}{$2000 / 01$} & Overall & 9050 & 279771 & 3.23 & 1079 & 141792 & 0.76 & 7971 & 137979 & 5.78 \\
\hline & 1 (lowest) & 2415 & 71731 & 3.37 & 329 & 36586 & 0.90 & 2086 & 35145 & 5.94 \\
\hline & 2 & 2095 & 64341 & 3.26 & 237 & 31397 & 0.75 & 1858 & 32944 & 5.64 \\
\hline & 3 & 1765 & 54645 & 3.23 & 206 & 27621 & 0.75 & 1559 & 27024 & 5.77 \\
\hline & 4 & 1426 & 46611 & 3.06 & 174 & 24421 & 0.71 & 1252 & 22190 & 5.64 \\
\hline & 5 (highest) & 1316 & 40328 & 3.26 & 127 & 20296 & 0.63 & 1189 & 20032 & 5.94 \\
\hline & Missing & 33 & 2115 & 1.56 & 6 & 1471 & 0.41 & 27 & 644 & 4.19 \\
\hline \multirow[t]{7}{*}{$2001 / 02$} & Overall & 9434 & 302975 & 3.11 & 1141 & 155172 & 0.74 & 8293 & 147803 & 5.61 \\
\hline & 1 (lowest) & 2453 & 76387 & 3.21 & 310 & 39263 & 0.79 & 2143 & 37124 & 5.77 \\
\hline & 2 & 2200 & 69239 & 3.18 & 258 & 34086 & 0.76 & 1942 & 35153 & 5.52 \\
\hline & 3 & 1872 & 59504 & 3.15 & 224 & 30400 & 0.74 & 1648 & 29104 & 5.66 \\
\hline & 4 & 1495 & 51436 & 2.91 & 182 & 27237 & 0.67 & 1313 & 24199 & 5.43 \\
\hline & 5 (highest) & 1365 & 44213 & 3.09 & 153 & 22674 & 0.67 & 1212 & 21539 & 5.63 \\
\hline & Missing & 49 & 2196 & 2.23 & 14 & 1512 & 0.93 & 35 & 684 & 5.12 \\
\hline \multirow[t]{7}{*}{$2002 / 03$} & Overall & 10204 & 327089 & 3.12 & 1272 & 169229 & 0.75 & 8932 & 157860 & 5.66 \\
\hline & 1 (lowest) & 2704 & 81010 & 3.34 & 386 & 41840 & 0.92 & 2318 & 39170 & 5.92 \\
\hline & 2 & 2386 & 74146 & 3.22 & 285 & 36850 & 0.77 & 2101 & 37296 & 5.63 \\
\hline & 3 & 1905 & 64506 & 2.95 & 209 & 33365 & 0.63 & 1696 & 31141 & 5.45 \\
\hline & 4 & 1621 & 56633 & 2.86 & 210 & 30343 & 0.69 & 1411 & 26290 & 5.37 \\
\hline & 5 (highest) & 1555 & 48450 & 3.21 & 175 & 25194 & 0.69 & 1380 & 23256 & 5.93 \\
\hline & Missing & 33 & 2344 & 1.41 & 7 & 1637 & 0.43 & 26 & 707 & 3.68 \\
\hline \multirow[t]{7}{*}{$2003 / 04$} & Overall & 10509 & 350520 & 3.00 & 1293 & 182997 & 0.71 & 9216 & 167523 & 5.50 \\
\hline & 1 (lowest) & 2717 & 84822 & 3.20 & 393 & 44077 & 0.89 & 2324 & 40745 & 5.70 \\
\hline & 2 & 2462 & 78866 & 3.12 & 278 & 39531 & 0.70 & 2184 & 39335 & 5.55 \\
\hline & 3 & 2065 & 69625 & 2.97 & 251 & 36372 & 0.69 & 1814 & 33253 & 5.46 \\
\hline & 4 & 1668 & 62183 & 2.68 & 207 & 33605 & 0.62 & 1461 & 28578 & 5.11 \\
\hline & 5 (highest) & 1551 & 52483 & 2.96 & 154 & 27648 & 0.56 & 1397 & 24835 & 5.63 \\
\hline & Missing & 46 & 2541 & 1.81 & 10 & 1764 & 0.57 & 36 & 777 & 4.63 \\
\hline \multirow[t]{7}{*}{$2004 / 05$} & Overall & 10798 & 377150 & 2.86 & 1251 & 198073 & 0.63 & 9547 & 179077 & 5.33 \\
\hline & 1 (lowest) & 2819 & 96675 & 2.92 & 402 & 51021 & 0.79 & 2417 & 45654 & 5.29 \\
\hline & 2 & 2408 & 84101 & 2.86 & 277 & 43423 & 0.64 & 2131 & 40678 & 5.24 \\
\hline & 3 & 2021 & 72443 & 2.79 & 224 & 38209 & 0.59 & 1797 & 34234 & 5.25 \\
\hline & 4 & 1832 & 65484 & 2.80 & 189 & 34741 & 0.54 & 1643 & 30743 & 5.34 \\
\hline & 5 (highest) & 1676 & 56501 & 2.97 & 150 & 29467 & 0.51 & 1526 & 27034 & 5.64 \\
\hline & Missing & 42 & 1946 & 2.16 & 9 & 1212 & 0.74 & 33 & 734 & 4.50 \\
\hline \multirow[t]{7}{*}{$2005 / 06$} & Overall & 11083 & 406829 & 2.72 & 1365 & 214312 & 0.64 & 9718 & 192517 & 5.05 \\
\hline & 1 (lowest) & 2937 & 102454 & 2.87 & 410 & 54172 & 0.76 & 2527 & 48282 & 5.23 \\
\hline & 2 & 2512 & 90459 & 2.78 & 341 & 46776 & 0.73 & 2171 & 43683 & 4.97 \\
\hline & 3 & 2136 & 79202 & 2.70 & 247 & 41915 & 0.59 & 1889 & 37287 & 5.07 \\
\hline & 4 & 1841 & 71387 & 2.58 & 200 & 37957 & 0.53 & 1641 & 33430 & 4.91 \\
\hline & 5 (highest) & 1608 & 61162 & 2.63 & 162 & 32146 & 0.50 & 1446 & 29016 & 4.98 \\
\hline & Missing & 49 & 2165 & 2.26 & 5 & 1346 & 0.37 & 44 & 819 & 5.37 \\
\hline
\end{tabular}


Table 3: Crude mortality data from $1994 / 95$ to 2005/06 among men with diabetes mellitus aged 30 years or more, by neighbourhood income and age group (part 1 of 2)

\begin{tabular}{|c|c|c|c|c|c|c|c|c|c|c|}
\hline \multirow[b]{2}{*}{ Year } & \multirow[b]{2}{*}{$\begin{array}{l}\text { Income } \\
\text { quintile }\end{array}$} & \multicolumn{3}{|c|}{ All ages ( $\geq 30 \mathrm{yr}$ ) } & \multicolumn{3}{|c|}{ Age 30-64 yr } & \multicolumn{3}{|c|}{ Age $\geq 65 \mathrm{yr}$} \\
\hline & & Deaths & Population & $\begin{array}{c}\text { Mortality, } \\
\%\end{array}$ & Deaths & Population & $\begin{array}{c}\text { Mortality, } \\
\%\end{array}$ & Deaths & Population & $\begin{array}{c}\text { Mortality, } \\
\%\end{array}$ \\
\hline \multirow[t]{7}{*}{$1994 / 95$} & Overall & 7979 & 190857 & 4.18 & 1620 & 108677 & 1.49 & 6359 & 82180 & 7.74 \\
\hline & 1 (lowest) & 1920 & 42630 & 4.50 & 409 & 24471 & 1.67 & 1511 & 18159 & 8.32 \\
\hline & 2 & 1758 & 41408 & 4.25 & 375 & 23376 & 1.60 & 1383 & 18032 & 7.67 \\
\hline & 3 & 1642 & 38907 & 4.22 & 306 & 21955 & 1.39 & 1336 & 16952 & 7.88 \\
\hline & 4 & 1328 & 34546 & 3.84 & 264 & 20060 & 1.32 & 1064 & 14486 & 7.35 \\
\hline & 5 (highest) & 1282 & 32064 & 4.00 & 251 & 17952 & 1.40 & 1031 & 14112 & 7.31 \\
\hline & Missing & 49 & 1302 & 3.76 & 15 & 863 & 1.74 & 34 & 439 & 7.74 \\
\hline \multirow[t]{7}{*}{$1995 / 96$} & Overall & 8534 & 207792 & 4.11 & 1694 & 118216 & 1.43 & 6840 & 89576 & 7.64 \\
\hline & 1 (lowest) & 2041 & 46199 & 4.42 & 435 & 26601 & 1.64 & 1606 & 19598 & 8.19 \\
\hline & 2 & 1934 & 44795 & 4.32 & 379 & 25225 & 1.50 & 1555 & 19570 & 7.95 \\
\hline & 3 & 1744 & 42191 & 4.13 & 332 & 23731 & 1.40 & 1412 & 18460 & 7.65 \\
\hline & 4 & 1443 & 38039 & 3.79 & 283 & 22094 & 1.28 & 1160 & 15945 & 7.28 \\
\hline & 5 (highest) & 1317 & 35191 & 3.74 & 248 & 19630 & 1.26 & 1069 & 15561 & 6.87 \\
\hline & Missing & 55 & 1377 & 3.99 & 17 & 935 & 1.82 & 38 & 442 & 8.60 \\
\hline \multirow[t]{7}{*}{$1996 / 97$} & Overall & 8981 & 224132 & 4.01 & 1663 & 127371 & 1.31 & 7318 & 96761 & 7.56 \\
\hline & 1 (lowest) & 2127 & 49258 & 4.32 & 441 & 28634 & 1.54 & 1686 & 20624 & 8.17 \\
\hline & 2 & 2027 & 47904 & 4.23 & 377 & 26849 & 1.40 & 1650 & 21055 & 7.84 \\
\hline & 3 & 1878 & 45621 & 4.12 & 335 & 25588 & 1.31 & 1543 & 20033 & 7.70 \\
\hline & 4 & 1509 & 41401 & 3.64 & 287 & 23896 & 1.20 & 1222 & 17505 & 6.98 \\
\hline & 5 (highest) & 1387 & 38375 & 3.61 & 211 & 21319 & 0.99 & 1176 & 17056 & 6.89 \\
\hline & Missing & 53 & 1573 & 3.37 & 12 & 1085 & 1.11 & 41 & 488 & 8.40 \\
\hline \multirow[t]{7}{*}{$1997 / 98$} & Overall & 9487 & 242424 & 3.91 & 1803 & 137885 & 1.31 & 7684 & 104539 & 7.35 \\
\hline & 1 (lowest) & 2242 & 52822 & 4.24 & 498 & 30941 & 1.61 & 1744 & 21881 & 7.97 \\
\hline & 2 & 2115 & 51551 & 4.10 & 390 & 28959 & 1.35 & 1725 & 22592 & 7.64 \\
\hline & 3 & 1954 & 49250 & 3.97 & 345 & 27615 & 1.25 & 1609 & 21635 & 7.44 \\
\hline & 4 & 1625 & 45281 & 3.59 & 304 & 26005 & 1.17 & 1321 & 19276 & 6.85 \\
\hline & 5 (highest) & 1504 & 41866 & 3.59 & 256 & 23222 & 1.10 & 1248 & 18644 & 6.69 \\
\hline & Missing & 47 & 1654 & 2.84 & 10 & 1143 & 0.87 & 37 & 511 & 7.24 \\
\hline \multirow[t]{7}{*}{$1998 / 99$} & Overall & 9858 & 260908 & 3.78 & 1815 & 148402 & 1.22 & 8043 & 112506 & 7.15 \\
\hline & 1 (lowest) & 2265 & 56371 & 4.02 & 480 & 33220 & 1.44 & 1785 & 23151 & 7.71 \\
\hline & 2 & 2181 & 54960 & 3.97 & 403 & 30912 & 1.30 & 1778 & 24048 & 7.39 \\
\hline & 3 & 1995 & 53051 & 3.76 & 351 & 29690 & 1.18 & 1644 & 23361 & 7.04 \\
\hline & 4 & 1782 & 49360 & 3.61 & 296 & 28369 & 1.04 & 1486 & 20991 & 7.08 \\
\hline & 5 (highest) & 1593 & 45331 & 3.51 & 276 & 24963 & 1.11 & 1317 & 20368 & 6.47 \\
\hline & Missing & 42 & 1835 & 2.29 & 9 & 1248 & 0.72 & 33 & 587 & 5.62 \\
\hline \multirow[t]{7}{*}{$1999 / 00$} & Overall & 10014 & 280980 & 3.56 & 1897 & 160109 & 1.18 & 8117 & 120871 & 6.72 \\
\hline & 1 (lowest) & 2445 & 62534 & 3.91 & 477 & 36516 & 1.31 & 1968 & 26018 & 7.56 \\
\hline & 2 & 2345 & 62057 & 3.78 & 410 & 34319 & 1.19 & 1935 & 27738 & 6.98 \\
\hline & 3 & 2044 & 56429 & 3.62 & 391 & 31800 & 1.23 & 1653 & 24629 & 6.71 \\
\hline & 4 & 1613 & 50485 & 3.20 & 335 & 29370 & 1.14 & 1278 & 21115 & 6.05 \\
\hline & 5 (highest) & 1525 & 47575 & 3.21 & 273 & 26776 & 1.02 & 1252 & 20799 & 6.02 \\
\hline & Missing & 42 & 1900 & 2.21 & 11 & 1328 & 0.83 & 31 & 572 & 5.42 \\
\hline
\end{tabular}


Table 3: Crude mortality data from $1994 / 95$ to 2005/06 among men with diabetes mellitus aged 30 years or more, by neighbourhood income and age group (part 2 of 2 )

\begin{tabular}{|c|c|c|c|c|c|c|c|c|c|c|}
\hline \multirow[b]{2}{*}{ Year } & \multirow[b]{2}{*}{$\begin{array}{l}\text { Income } \\
\text { quintile }\end{array}$} & \multicolumn{3}{|c|}{ All ages ( $\geq 30 \mathrm{yr}$ ) } & \multicolumn{3}{|c|}{ Age $30-64$ yr } & \multicolumn{3}{|c|}{ Age $\geq 65 \mathrm{yr}$} \\
\hline & & Deaths & Population & $\begin{array}{c}\text { Mortality, } \\
\%\end{array}$ & Deaths & Population & $\begin{array}{c}\text { Mortality, } \\
\%\end{array}$ & Deaths & Population & $\begin{array}{c}\text { Mortality, } \\
\%\end{array}$ \\
\hline \multirow[t]{7}{*}{$2000 / 01$} & Overall & 10491 & 302342 & 3.47 & 1957 & 172513 & 1.13 & 8534 & 129829 & 6.57 \\
\hline & 1 (lowest) & 2568 & 66652 & 3.85 & 555 & 38958 & 1.42 & 2013 & 27694 & 7.27 \\
\hline & 2 & 2446 & 66372 & 3.69 & 431 & 36800 & 1.17 & 2015 & 29572 & 6.81 \\
\hline & 3 & 2091 & 60874 & 3.43 & 353 & 34463 & 1.02 & 1738 & 26411 & 6.58 \\
\hline & 4 & 1656 & 55063 & 3.01 & 343 & 32010 & 1.07 & 1313 & 23053 & 5.70 \\
\hline & 5 (highest) & 1692 & 51413 & 3.29 & 262 & 28900 & 0.91 & 1430 & 22513 & 6.35 \\
\hline & Missing & 38 & 1968 & 1.93 & 13 & 1382 & 0.94 & 25 & 586 & 4.27 \\
\hline \multirow[t]{7}{*}{$2001 / 02$} & Overall & 10841 & 327318 & 3.31 & 2052 & 187404 & 1.09 & 8789 & 139914 & 6.28 \\
\hline & 1 (lowest) & 2606 & 71305 & 3.65 & 554 & 41685 & 1.33 & 2052 & 29620 & 6.93 \\
\hline & 2 & 2552 & 71614 & 3.56 & 462 & 39826 & 1.16 & 2090 & 31788 & 6.57 \\
\hline & 3 & 2085 & 65736 & 3.17 & 370 & 37552 & 0.99 & 1715 & 28184 & 6.09 \\
\hline & 4 & 1804 & 60531 & 2.98 & 370 & 35220 & 1.05 & 1434 & 25311 & 5.67 \\
\hline & 5 (highest) & 1744 & 56010 & 3.11 & 276 & 31646 & 0.87 & 1468 & 24364 & 6.03 \\
\hline & Missing & 50 & 2122 & 2.36 & 20 & 1475 & 1.36 & 30 & 647 & 4.64 \\
\hline \multirow[t]{7}{*}{$2002 / 03$} & Overall & 11415 & 353188 & 3.23 & 2149 & 202776 & 1.06 & 9266 & 150412 & 6.16 \\
\hline & 1 (lowest) & 2738 & 75560 & 3.62 & 596 & 44252 & 1.35 & 2142 & 31308 & 6.84 \\
\hline & 2 & 2642 & 76743 & 3.44 & 477 & 42889 & 1.11 & 2165 & 33854 & 6.40 \\
\hline & 3 & 2290 & 71350 & 3.21 & 404 & 40777 & 0.99 & 1886 & 30573 & 6.17 \\
\hline & 4 & 1882 & 66464 & 2.83 & 355 & 38917 & 0.91 & 1527 & 27547 & 5.54 \\
\hline & 5 (highest) & 1812 & 60801 & 2.98 & 304 & 34355 & 0.88 & 1508 & 26446 & 5.70 \\
\hline & Missing & 51 & 2270 & 2.25 & 13 & 1586 & 0.82 & 38 & 684 & 5.56 \\
\hline \multirow[t]{7}{*}{$2003 / 04$} & Overall & 12169 & 377557 & 3.22 & 2303 & 217002 & 1.06 & 9866 & 160555 & 6.14 \\
\hline & 1 (lowest) & 2810 & 79196 & 3.55 & 602 & 46422 & 1.30 & 2208 & 32774 & 6.74 \\
\hline & 2 & 2790 & 81123 & 3.44 & 540 & 45319 & 1.19 & 2250 & 35804 & 6.28 \\
\hline & 3 & 2459 & 76763 & 3.20 & 441 & 44008 & 1.00 & 2018 & 32755 & 6.16 \\
\hline & 4 & 2195 & 72409 & 3.03 & 394 & 42344 & 0.93 & 1801 & 30065 & 5.99 \\
\hline & 5 (highest) & 1858 & 65533 & 2.84 & 309 & 37164 & 0.83 & 1549 & 28369 & 5.46 \\
\hline & Missing & 57 & 2533 & 2.25 & 17 & 1745 & 0.97 & 40 & 788 & 5.08 \\
\hline \multirow[t]{7}{*}{$2004 / 05$} & Overall & 12179 & 405183 & 3.01 & 2300 & 232994 & 0.99 & 9879 & 172189 & 5.74 \\
\hline & 1 (lowest) & 2923 & 90606 & 3.23 & 637 & 53894 & 1.18 & 2286 & 36712 & 6.23 \\
\hline & 2 & 2695 & 87798 & 3.07 & 493 & 49927 & 0.99 & 2202 & 37871 & 5.81 \\
\hline & 3 & 2325 & 79481 & 2.93 & 429 & 45590 & 0.94 & 1896 & 33891 & 5.59 \\
\hline & 4 & 2122 & 76098 & 2.79 & 384 & 43476 & 0.88 & 1738 & 32622 & 5.33 \\
\hline & 5 (highest) & 2060 & 69001 & 2.99 & 342 & 38650 & 0.88 & 1718 & 30351 & 5.66 \\
\hline & Missing & 54 & 2199 & 2.46 & 15 & 1457 & 1.03 & 39 & 742 & 5.26 \\
\hline \multirow[t]{7}{*}{$2005 / 06$} & Overall & 12469 & 436800 & 2.85 & 2284 & 251069 & 0.91 & 10185 & 185731 & 5.48 \\
\hline & 1 (lowest) & 2993 & 96019 & 3.12 & 652 & 57004 & 1.14 & 2341 & 39015 & 6.00 \\
\hline & 2 & 2779 & 94278 & 2.95 & 486 & 53714 & 0.90 & 2293 & 40564 & 5.65 \\
\hline & 3 & 2411 & 86628 & 2.78 & 431 & 49741 & 0.87 & 1980 & 36887 & 5.37 \\
\hline & 4 & 2228 & 82824 & 2.69 & 398 & 47391 & 0.84 & 1830 & 35433 & 5.16 \\
\hline & 5 (highest) & 2012 & 74561 & 2.70 & 309 & 41573 & 0.74 & 1703 & 32988 & 5.16 \\
\hline & Missing & 46 & 2490 & 1.85 & 8 & 1646 & 0.49 & 38 & 844 & 4.50 \\
\hline
\end{tabular}


Table 4: Crude mortality data from 1994/95 to 2005/06 among people without diabetes aged 30 years or more, by neighbourhood income and age group (part 1 of 2)

\begin{tabular}{|c|c|c|c|c|c|c|c|c|c|c|}
\hline \multirow[b]{2}{*}{ Year } & \multirow[b]{2}{*}{$\begin{array}{l}\text { Income } \\
\text { quintile }\end{array}$} & \multicolumn{3}{|c|}{ All ages ( $\geq 30 \mathrm{yr}$ ) } & \multicolumn{3}{|c|}{ Age $30-64$ yr } & \multicolumn{3}{|c|}{ Age $\geq 65 \mathrm{yr}$} \\
\hline & & Deaths & Population & $\begin{array}{c}\text { Mortality, } \\
\%\end{array}$ & Deaths & Population & $\begin{array}{c}\text { Mortality, } \\
\%\end{array}$ & Deaths & Population & $\begin{array}{c}\text { Mortality, } \\
\%\end{array}$ \\
\hline \multirow[t]{6}{*}{$1994 / 95$} & Overall & 58591 & 5893668 & 0.99 & 12694 & 4771138 & 0.27 & 45897 & 1122530 & 4.09 \\
\hline & 1 (lowest) & 13656 & 1107058 & 1.23 & 3042 & 875975 & 0.35 & 10614 & 231083 & 4.59 \\
\hline & 2 & 12669 & 1161176 & 1.09 & 2631 & 922108 & 0.29 & 10038 & 239068 & 4.20 \\
\hline & 3 & 11701 & 1184150 & 0.99 & 2513 & 957241 & 0.26 & 9188 & 226909 & 4.05 \\
\hline & 4 & 10200 & 1205792 & 0.85 & 2315 & 1001017 & 0.23 & 7885 & 204775 & 3.85 \\
\hline & 5 (highest) & 10365 & 1235492 & 0.84 & 2193 & 1014797 & 0.22 & 8172 & 220695 & 3.70 \\
\hline \multirow[t]{6}{*}{$1995 / 96$} & Overall & 59060 & 6000780 & 0.98 & 12803 & 4864309 & 0.26 & 46257 & 1136471 & 4.07 \\
\hline & 1 (lowest) & 13636 & 1123834 & 1.21 & 3078 & 890260 & 0.35 & 10558 & 233574 & 4.52 \\
\hline & 2 & 12869 & 1180794 & 1.09 & 2744 & 939023 & 0.29 & 10125 & 241771 & 4.19 \\
\hline & 3 & 11740 & 1206103 & 0.97 & 2461 & 976307 & 0.25 & 9279 & 229796 & 4.04 \\
\hline & 4 & 10273 & 1229136 & 0.84 & 2274 & 1021638 & 0.22 & 7999 & 207498 & 3.85 \\
\hline & 5 (highest) & 10542 & 1260913 & 0.84 & 2246 & 1037081 & 0.22 & 8296 & 223832 & 3.71 \\
\hline \multirow[t]{6}{*}{$1996 / 97$} & Overall & 58844 & 6097842 & 0.96 & 12444 & 4946766 & 0.25 & 46400 & 1151076 & 4.03 \\
\hline & 1 (lowest) & 13640 & 1138989 & 1.20 & 3027 & 902531 & 0.34 & 10613 & 236458 & 4.49 \\
\hline & 2 & 12455 & 1198355 & 1.04 & 2549 & 953695 & 0.27 & 9906 & 244660 & 4.05 \\
\hline & 3 & 11870 & 1225301 & 0.97 & 2390 & 992689 & 0.24 & 9480 & 232612 & 4.08 \\
\hline & 4 & 10209 & 1250212 & 0.82 & 2271 & 1039969 & 0.22 & 7938 & 210243 & 3.78 \\
\hline & 5 (highest) & 10670 & 1284985 & 0.83 & 2207 & 1057882 & 0.21 & 8463 & 227103 & 3.73 \\
\hline \multirow[t]{6}{*}{$1997 / 98$} & Overall & 58336 & 6192119 & 0.94 & 12011 & 5027549 & 0.24 & 46325 & 1164570 & 3.98 \\
\hline & 1 (lowest) & 13343 & 1151432 & 1.16 & 2812 & 911996 & 0.31 & 10531 & 239436 & 4.40 \\
\hline & 2 & 12342 & 1214383 & 1.02 & 2501 & 967109 & 0.26 & 9841 & 247274 & 3.98 \\
\hline & 3 & 11939 & 1244785 & 0.96 & 2412 & 1009642 & 0.24 & 9527 & 235143 & 4.05 \\
\hline & 4 & 10301 & 1271871 & 0.81 & 2202 & 1059331 & 0.21 & 8099 & 212540 & 3.81 \\
\hline & 5 (highest) & 10411 & 1309648 & 0.79 & 2084 & 1079471 & 0.19 & 8327 & 230177 & 3.62 \\
\hline \multirow[t]{6}{*}{$1998 / 99$} & Overall & 58021 & 6274810 & 0.92 & 11614 & 5098397 & 0.23 & 46407 & 1176413 & 3.94 \\
\hline & 1 (lowest) & 13189 & 1160019 & 1.14 & 2785 & 918455 & 0.30 & 10404 & 241564 & 4.31 \\
\hline & 2 & 12570 & 1227671 & 1.02 & 2454 & 978080 & 0.25 & 10116 & 249591 & 4.05 \\
\hline & 3 & 11861 & 1262253 & 0.94 & 2255 & 1024623 & 0.22 & 9606 & 237630 & 4.04 \\
\hline & 4 & 10210 & 1291781 & 0.79 & 2113 & 1077001 & 0.20 & 8097 & 214780 & 3.77 \\
\hline & 5 (highest) & 10191 & 1333086 & 0.76 & 2007 & 1100238 & 0.18 & 8184 & 232848 & 3.51 \\
\hline \multirow[t]{6}{*}{$1999 / 00$} & Overall & 58786 & 6356146 & 0.92 & 11690 & 5171727 & 0.23 & 47096 & 1184419 & 3.98 \\
\hline & 1 (lowest) & 14493 & 1199198 & 1.21 & 2914 & 954696 & 0.31 & 11579 & 244502 & 4.74 \\
\hline & 2 & 13088 & 1259662 & 1.04 & 2522 & 1003096 & 0.25 & 10566 & 256566 & 4.12 \\
\hline & 3 & 11435 & 1282123 & 0.89 & 2252 & 1046467 & 0.22 & 9183 & 235656 & 3.90 \\
\hline & 4 & 9604 & 1293188 & 0.74 & 2062 & 1077147 & 0.19 & 7542 & 216041 & 3.49 \\
\hline & 5 (highest) & 10166 & 1321975 & 0.77 & 1940 & 1090321 & 0.18 & 8226 & 231654 & 3.55 \\
\hline
\end{tabular}


Table 4: Crude mortality data from $1994 / 95$ to 2005/06 among people without diabetes aged 30 years or more, by neighbourhood income and age group (part 2 of 2)

\begin{tabular}{|c|c|c|c|c|c|c|c|c|c|c|}
\hline \multirow[b]{2}{*}{ Year } & \multirow[b]{2}{*}{$\begin{array}{l}\text { Income } \\
\text { quintile }\end{array}$} & \multicolumn{3}{|c|}{ All ages ( $\geq 30 \mathrm{yr}$ ) } & \multicolumn{3}{|c|}{ Age $30-64$ yr } & \multicolumn{3}{|c|}{ Age $\geq 65 y r$} \\
\hline & & Deaths & Population & $\begin{array}{c}\text { Mortality, } \\
\%\end{array}$ & Deaths & Population & $\begin{array}{c}\text { Mortality, } \\
\%\end{array}$ & Deaths & Population & $\begin{array}{c}\text { Mortality, } \\
\%\end{array}$ \\
\hline \multirow[t]{6}{*}{$2000 / 01$} & Overall & 58194 & 6450421 & 0.90 & 11700 & 5258319 & 0.22 & 46494 & 1192102 & 3.90 \\
\hline & 1 (lowest) & 13814 & 1209323 & 1.14 & 2813 & 963618 & 0.29 & 11001 & 245705 & 4.48 \\
\hline & 2 & 12912 & 1275352 & 1.01 & 2523 & 1017319 & 0.25 & 10389 & 258033 & 4.03 \\
\hline & 3 & 11714 & 1301059 & 0.90 & 2320 & 1063869 & 0.22 & 9394 & 237190 & 3.96 \\
\hline & 4 & 9797 & 1316605 & 0.74 & 2095 & 1099219 & 0.19 & 7702 & 217386 & 3.54 \\
\hline & 5 (highest) & 9957 & 1348082 & 0.74 & 1949 & 1114294 & 0.17 & 8008 & 233788 & 3.43 \\
\hline \multirow[t]{6}{*}{$2001 / 02$} & Overall & 57424 & 6554600 & 0.88 & 11632 & 5355002 & 0.22 & 45792 & 1199598 & 3.82 \\
\hline & 1 (lowest) & 13731 & 1223106 & 1.12 & 2805 & 976592 & 0.29 & 10926 & 246514 & 4.43 \\
\hline & 2 & 12838 & 1293336 & 0.99 & 2611 & 1033966 & 0.25 & 10227 & 259370 & 3.94 \\
\hline & 3 & 11301 & 1322131 & 0.85 & 2191 & 1083253 & 0.20 & 9110 & 238878 & 3.81 \\
\hline & 4 & 9386 & 1340735 & 0.70 & 2011 & 1122022 & 0.18 & 7375 & 218713 & 3.37 \\
\hline & 5 (highest) & 10168 & 1375292 & 0.74 & 2014 & 1139169 & 0.18 & 8154 & 236123 & 3.45 \\
\hline \multirow[t]{6}{*}{$2002 / 03$} & Overall & 57092 & 6661144 & 0.86 & 11954 & 5451319 & 0.22 & 45138 & 1209825 & 3.73 \\
\hline & 1 (lowest) & 13352 & 1234283 & 1.08 & 2854 & 986385 & 0.29 & 10498 & 247898 & 4.23 \\
\hline & 2 & 12687 & 1311262 & 0.97 & 2485 & 1049657 & 0.24 & 10202 & 261605 & 3.90 \\
\hline & 3 & 11208 & 1343912 & 0.83 & 2335 & 1103201 & 0.21 & 8873 & 240711 & 3.69 \\
\hline & 4 & 9758 & 1367638 & 0.71 & 2186 & 1146806 & 0.19 & 7572 & 220832 & 3.43 \\
\hline & 5 (highest) & 10087 & 1404049 & 0.72 & 2094 & 1165270 & 0.18 & 7993 & 238779 & 3.35 \\
\hline \multirow[t]{6}{*}{$2003 / 04$} & Overall & 58533 & 6745633 & 0.87 & 12384 & 5525278 & 0.22 & 46149 & 1220355 & 3.78 \\
\hline & 1 (lowest) & 13375 & 1241299 & 1.08 & 2924 & 991477 & 0.29 & 10451 & 249822 & 4.18 \\
\hline & 2 & 12963 & 1325056 & 0.98 & 2670 & 1061126 & 0.25 & 10293 & 263930 & 3.90 \\
\hline & 3 & 11539 & 1360984 & 0.85 & 2403 & 1118385 & 0.21 & 9136 & 242599 & 3.77 \\
\hline & 4 & 10202 & 1389481 & 0.73 & 2205 & 1167094 & 0.19 & 7997 & 222387 & 3.60 \\
\hline & 5 (highest) & 10454 & 1428813 & 0.73 & 2182 & 1187196 & 0.18 & 8272 & 241617 & 3.42 \\
\hline \multirow[t]{6}{*}{$2004 / 05$} & Overall & 57044 & 6826813 & 0.84 & 12134 & 5595432 & 0.22 & 44910 & 1231381 & 3.65 \\
\hline & 1 (lowest) & 13527 & 1270203 & 1.06 & 2954 & 1030059 & 0.29 & 10573 & 240144 & 4.40 \\
\hline & 2 & 12174 & 1353483 & 0.90 & 2667 & 1095356 & 0.24 & 9507 & 258127 & 3.68 \\
\hline & 3 & 10858 & 1364921 & 0.80 & 2323 & 1124005 & 0.21 & 8535 & 240916 & 3.54 \\
\hline & 4 & 10333 & 1409504 & 0.73 & 2161 & 1170041 & 0.18 & 8172 & 239463 & 3.41 \\
\hline & 5 (highest) & 10152 & 1428702 & 0.71 & 2029 & 1175971 & 0.17 & 8123 & 252731 & 3.21 \\
\hline \multirow[t]{6}{*}{$2005 / 06$} & Overall & 58953 & 6888074 & 0.86 & 12294 & 5656014 & 0.22 & 46659 & 1232060 & 3.79 \\
\hline & 1 (lowest) & 13725 & 1273696 & 1.08 & 3216 & 1034327 & 0.31 & 10509 & 239369 & 4.39 \\
\hline & 2 & 12535 & 1361984 & 0.92 & 2559 & 1104300 & 0.23 & 9976 & 257684 & 3.87 \\
\hline & 3 & 11164 & 1377577 & 0.81 & 2267 & 1136832 & 0.20 & 8897 & 240745 & 3.70 \\
\hline & 4 & 10790 & 1426340 & 0.76 & 2194 & 1186161 & 0.18 & 8596 & 240179 & 3.58 \\
\hline & 5 (highest) & 10739 & 1448477 & 0.74 & 2058 & 1194394 & 0.17 & 8681 & 254083 & 3.42 \\
\hline
\end{tabular}


Table 5: Crude mortality data from 1994/95 to 2005/06 among women without diabetes aged 30 years or more, by neighbourhood income and age group (part 1 of 2)

\begin{tabular}{|c|c|c|c|c|c|c|c|c|c|c|}
\hline \multirow[b]{2}{*}{ Year } & \multirow[b]{2}{*}{$\begin{array}{l}\text { Income } \\
\text { quintile }\end{array}$} & \multicolumn{3}{|c|}{ All ages ( $\geq 30 \mathrm{yr}$ ) } & \multicolumn{3}{|c|}{ Age 30-64 yr } & \multicolumn{3}{|c|}{ Age $\geq 65 \mathrm{yr}$} \\
\hline & & Deaths & Population & $\begin{array}{c}\text { Mortality, } \\
\%\end{array}$ & Deaths & Population & $\begin{array}{c}\text { Mortality, } \\
\%\end{array}$ & Deaths & Population & $\begin{array}{c}\text { Mortality, } \\
\%\end{array}$ \\
\hline \multirow[t]{6}{*}{$1994 / 95$} & Overall & 28779 & 3072773 & 0.94 & 4633 & 2413087 & 0.19 & 24146 & 659686 & 3.66 \\
\hline & 1 (lowest) & 6760 & 585037 & 1.16 & 1061 & 442632 & 0.24 & 5699 & 142405 & 4.00 \\
\hline & 2 & 6188 & 608498 & 1.02 & 950 & 465799 & 0.20 & 5238 & 142699 & 3.67 \\
\hline & 3 & 5698 & 617515 & 0.92 & 899 & 484212 & 0.19 & 4799 & 133303 & 3.60 \\
\hline & 4 & 4978 & 621407 & 0.80 & 873 & 504413 & 0.17 & 4105 & 116994 & 3.51 \\
\hline & 5 (highest) & 5155 & 640316 & 0.81 & 850 & 516031 & 0.16 & 4305 & 124285 & 3.46 \\
\hline \multirow[t]{6}{*}{ 1995/96 } & Overall & 29165 & 3130417 & 0.93 & 4817 & 2463145 & 0.20 & 24348 & 667272 & 3.65 \\
\hline & 1 (lowest) & 6701 & 594146 & 1.13 & 1096 & 450200 & 0.24 & 5605 & 143946 & 3.89 \\
\hline & 2 & 6389 & 619007 & 1.03 & 1050 & 474810 & 0.22 & 5339 & 144197 & 3.70 \\
\hline & 3 & 5796 & 629232 & 0.92 & 931 & 494388 & 0.19 & 4865 & 134844 & 3.61 \\
\hline & 4 & 5052 & 633911 & 0.80 & 869 & 515481 & 0.17 & 4183 & 118430 & 3.53 \\
\hline & 5 (highest) & 5227 & 654121 & 0.80 & 871 & 528266 & 0.16 & 4356 & 125855 & 3.46 \\
\hline \multirow[t]{6}{*}{ 1996/97 } & Overall & 29187 & 3182141 & 0.92 & 4779 & 2507085 & 0.19 & 24408 & 675056 & 3.62 \\
\hline & 1 (lowest) & 6717 & 601995 & 1.12 & 1074 & 456547 & 0.24 & 5643 & 145448 & 3.88 \\
\hline & 2 & 6163 & 628335 & 0.98 & 961 & 482521 & 0.20 & 5202 & 145814 & 3.57 \\
\hline & 3 & 5970 & 639484 & 0.93 & 952 & 503153 & 0.19 & 5018 & 136331 & 3.68 \\
\hline & 4 & 4962 & 645236 & 0.77 & 898 & 525322 & 0.17 & 4064 & 119914 & 3.39 \\
\hline & 5 (highest) & 5375 & 667091 & 0.81 & 894 & 539542 & 0.17 & 4481 & 127549 & 3.51 \\
\hline \multirow[t]{6}{*}{$1997 / 98$} & Overall & 29243 & 3229255 & 0.91 & 4658 & 2547669 & 0.18 & 24585 & 681586 & 3.61 \\
\hline & 1 (lowest) & 6851 & 608294 & 1.13 & 1094 & 461135 & 0.24 & 5757 & 147159 & 3.91 \\
\hline & 2 & 6196 & 636344 & 0.97 & 948 & 489271 & 0.19 & 5248 & 147073 & 3.57 \\
\hline & 3 & 5947 & 649105 & 0.92 & 918 & 511713 & 0.18 & 5029 & 137392 & 3.66 \\
\hline & 4 & 5033 & 656016 & 0.77 & 856 & 535011 & 0.16 & 4177 & 121005 & 3.45 \\
\hline & 5 (highest) & 5216 & 679496 & 0.77 & 842 & 550539 & 0.15 & 4374 & 128957 & 3.39 \\
\hline \multirow[t]{6}{*}{ 1998/99 } & Overall & 29287 & 3270017 & 0.90 & 4609 & 2583052 & 0.18 & 24678 & 686965 & 3.59 \\
\hline & 1 (lowest) & 6815 & 612700 & 1.11 & 1080 & 464596 & 0.23 & 5735 & 148104 & 3.87 \\
\hline & 2 & 6342 & 642818 & 0.99 & 941 & 494804 & 0.19 & 5401 & 148014 & 3.65 \\
\hline & 3 & 6027 & 657775 & 0.92 & 916 & 519167 & 0.18 & 5111 & 138608 & 3.69 \\
\hline & 4 & 4990 & 665749 & 0.75 & 844 & 543709 & 0.16 & 4146 & 122040 & 3.40 \\
\hline & 5 (highest) & 5113 & 690975 & 0.74 & 828 & 560776 & 0.15 & 4285 & 130199 & 3.29 \\
\hline \multirow[t]{6}{*}{$1999 / 00$} & Overall & 29755 & 3310438 & 0.90 & 4644 & 2619807 & 0.18 & 25111 & 690631 & 3.64 \\
\hline & 1 (lowest) & 7464 & 633315 & 1.18 & 1080 & 482275 & 0.22 & 6384 & 151040 & 4.23 \\
\hline & 2 & 6650 & 660366 & 1.01 & 989 & 507410 & 0.19 & 5661 & 152956 & 3.70 \\
\hline & 3 & 5798 & 666125 & 0.87 & 915 & 529506 & 0.17 & 4883 & 136619 & 3.57 \\
\hline & 4 & 4677 & 666936 & 0.70 & 817 & 545140 & 0.15 & 3860 & 121796 & 3.17 \\
\hline & 5 (highest) & 5166 & 683696 & 0.76 & 843 & 555476 & 0.15 & 4323 & 128220 & 3.37 \\
\hline
\end{tabular}


Table 5: Crude mortality data from $1994 / 95$ to 2005/06 among women without diabetes aged 30 years or more, by neighbourhood income and age group (part 2 of 2)

\begin{tabular}{|c|c|c|c|c|c|c|c|c|c|c|}
\hline \multirow[b]{2}{*}{ Year } & \multirow[b]{2}{*}{$\begin{array}{l}\text { Income } \\
\text { quintile }\end{array}$} & \multicolumn{3}{|c|}{ All ages ( $\geq 30 \mathrm{yr}$ ) } & \multicolumn{3}{|c|}{ Age $30-64 \mathrm{yr}$} & \multicolumn{3}{|c|}{ Age $\geq 65 \mathrm{yr}$} \\
\hline & & Deaths & Population & $\begin{array}{c}\text { Mortality, } \\
\%\end{array}$ & Deaths & Population & $\begin{array}{c}\text { Mortality, } \\
\%\end{array}$ & Deaths & Population & $\begin{array}{c}\text { Mortality, } \\
\%\end{array}$ \\
\hline \multirow[t]{6}{*}{$2000 / 01$} & Overall & 29670 & 3356643 & 0.88 & 4602 & 2662351 & 0.17 & 25068 & 694292 & 3.61 \\
\hline & 1 (lowest) & 7265 & 638114 & 1.14 & 1077 & 486297 & 0.22 & 6188 & 151817 & 4.08 \\
\hline & 2 & 6669 & 668028 & 1.00 & 966 & 514441 & 0.19 & 5703 & 153587 & 3.71 \\
\hline & 3 & 5824 & 675391 & 0.86 & 911 & 538106 & 0.17 & 4913 & 137285 & 3.58 \\
\hline & 4 & 4809 & 678464 & 0.71 & 842 & 556082 & 0.15 & 3967 & 122382 & 3.24 \\
\hline & 5 (highest) & 5103 & 696646 & 0.73 & 806 & 567425 & 0.14 & 4297 & 129221 & 3.33 \\
\hline \multirow[t]{6}{*}{$2001 / 02$} & Overall & 57424 & 6554600 & 0.88 & 11632 & 5355002 & 0.22 & 45792 & 1199598 & 3.82 \\
\hline & 1 (lowest) & 13731 & 1223106 & 1.12 & 2805 & 976592 & 0.29 & 10926 & 246514 & 4.43 \\
\hline & 2 & 12838 & 1293336 & 0.99 & 2611 & 1033966 & 0.25 & 10227 & 259370 & 3.94 \\
\hline & 3 & 11301 & 1322131 & 0.85 & 2191 & 1083253 & 0.20 & 9110 & 238878 & 3.81 \\
\hline & 4 & 9386 & 1340735 & 0.70 & 2011 & 1122022 & 0.18 & 7375 & 218713 & 3.37 \\
\hline & 5 (highest) & 10168 & 1375292 & 0.74 & 2014 & 1139169 & 0.18 & 8154 & 236123 & 3.45 \\
\hline \multirow[t]{6}{*}{$2002 / 03$} & Overall & 57092 & 6661144 & 0.86 & 11954 & 5451319 & 0.22 & 45138 & 1209825 & 3.73 \\
\hline & 1 (lowest) & 13352 & 1234283 & 1.08 & 2854 & 986385 & 0.29 & 10498 & 247898 & 4.23 \\
\hline & 2 & 12687 & 1311262 & 0.97 & 2485 & 1049657 & 0.24 & 10202 & 261605 & 3.90 \\
\hline & 3 & 11208 & 1343912 & 0.83 & 2335 & 1103201 & 0.21 & 8873 & 240711 & 3.69 \\
\hline & 4 & 9758 & 1367638 & 0.71 & 2186 & 1146806 & 0.19 & 7572 & 220832 & 3.43 \\
\hline & 5 (highest) & 10087 & 1404049 & 0.72 & 2094 & 1165270 & 0.18 & 7993 & 238779 & 3.35 \\
\hline \multirow[t]{6}{*}{$2003 / 04$} & Overall & 58533 & 6745633 & 0.87 & 12384 & 5525278 & 0.22 & 46149 & 1220355 & 3.78 \\
\hline & 1 (lowest) & 13375 & 1241299 & 1.08 & 2924 & 991477 & 0.29 & 10451 & 249822 & 4.18 \\
\hline & 2 & 12963 & 1325056 & 0.98 & 2670 & 1061126 & 0.25 & 10293 & 263930 & 3.90 \\
\hline & 3 & 11539 & 1360984 & 0.85 & 2403 & 1118385 & 0.21 & 9136 & 242599 & 3.77 \\
\hline & 4 & 10202 & 1389481 & 0.73 & 2205 & 1167094 & 0.19 & 7997 & 222387 & 3.60 \\
\hline & 5 (highest) & 10454 & 1428813 & 0.73 & 2182 & 1187196 & 0.18 & 8272 & 241617 & 3.42 \\
\hline \multirow[t]{6}{*}{$2004 / 05$} & Overall & 57044 & 6826813 & 0.84 & 12134 & 5595432 & 0.22 & 44910 & 1231381 & 3.65 \\
\hline & 1 (lowest) & 13527 & 1270203 & 1.06 & 2954 & 1030059 & 0.29 & 10573 & 240144 & 4.40 \\
\hline & 2 & 12174 & 1353483 & 0.90 & 2667 & 1095356 & 0.24 & 9507 & 258127 & 3.68 \\
\hline & 3 & 10858 & 1364921 & 0.80 & 2323 & 1124005 & 0.21 & 8535 & 240916 & 3.54 \\
\hline & 4 & 10333 & 1409504 & 0.73 & 2161 & 1170041 & 0.18 & 8172 & 239463 & 3.41 \\
\hline & 5 (highest) & 10152 & 1428702 & 0.71 & 2029 & 1175971 & 0.17 & 8123 & 252731 & 3.21 \\
\hline \multirow[t]{6}{*}{$2005 / 06$} & Overall & 58953 & 6888074 & 0.86 & 12294 & 5656014 & 0.22 & 46659 & 1232060 & 3.79 \\
\hline & 1 (lowest) & 13725 & 1273696 & 1.08 & 3216 & 1034327 & 0.31 & 10509 & 239369 & 4.39 \\
\hline & 2 & 12535 & 1361984 & 0.92 & 2559 & 1104300 & 0.23 & 9976 & 257684 & 3.87 \\
\hline & 3 & 11164 & 1377577 & 0.81 & 2267 & 1136832 & 0.20 & 8897 & 240745 & 3.70 \\
\hline & 4 & 10790 & 1426340 & 0.76 & 2194 & 1186161 & 0.18 & 8596 & 240179 & 3.58 \\
\hline & 5 (highest) & 10739 & 1448477 & 0.74 & 2058 & 1194394 & 0.17 & 8681 & 254083 & 3.42 \\
\hline
\end{tabular}


Table 6: Crude mortality data from 1994/95 to 2005/06 among men without diabetes aged 30 years or more, by neighbourhood income and age group (part 1 of 2)

\begin{tabular}{|c|c|c|c|c|c|c|c|c|c|c|}
\hline \multirow[b]{2}{*}{ Year } & \multirow[b]{2}{*}{$\begin{array}{l}\text { Income } \\
\text { quintile }\end{array}$} & \multicolumn{3}{|c|}{ All ages ( $\geq 30 y r)$} & \multicolumn{3}{|c|}{ Age $30-64$ yr } & \multicolumn{3}{|c|}{ Age $\geq 65 \mathrm{yr}$} \\
\hline & & Deaths & Population & $\begin{array}{c}\text { Mortality, } \\
\%\end{array}$ & Deaths & Population & $\begin{array}{c}\text { Mortality, } \\
\%\end{array}$ & Deaths & Population & $\begin{array}{c}\text { Mortality, } \\
\%\end{array}$ \\
\hline \multirow[t]{6}{*}{$1994 / 95$} & Overall & 29812 & 2820895 & 1.06 & 8061 & 2358051 & 0.34 & 21751 & 462844 & 4.70 \\
\hline & 1 (lowest) & 6896 & 522021 & 1.32 & 1981 & 433343 & 0.46 & 4915 & 88678 & 5.54 \\
\hline & 2 & 6481 & 552678 & 1.17 & 1681 & 456309 & 0.37 & 4800 & 96369 & 4.98 \\
\hline & 3 & 6003 & 566635 & 1.06 & 1614 & 473029 & 0.34 & 4389 & 93606 & 4.69 \\
\hline & 4 & 5222 & 584385 & 0.89 & 1442 & 496604 & 0.29 & 3780 & 87781 & 4.31 \\
\hline & 5 (highest) & 5210 & 595176 & 0.88 & 1343 & 498766 & 0.27 & 3867 & 96410 & 4.01 \\
\hline \multirow[t]{6}{*}{$1995 / 96$} & Overall & 29895 & 2870363 & 1.04 & 7986 & 2401164 & 0.33 & 21909 & 469199 & 4.67 \\
\hline & 1 (lowest) & 6935 & 529688 & 1.31 & 1982 & 440060 & 0.45 & 4953 & 89628 & 5.53 \\
\hline & 2 & 6480 & 561787 & 1.15 & 1694 & 464213 & 0.36 & 4786 & 97574 & 4.90 \\
\hline & 3 & 5944 & 576871 & 1.03 & 1530 & 481919 & 0.32 & 4414 & 94952 & 4.65 \\
\hline & 4 & 5221 & 595225 & 0.88 & 1405 & 506157 & 0.28 & 3816 & 89068 & 4.28 \\
\hline & 5 (highest) & 5315 & 606792 & 0.88 & 1375 & 508815 & 0.27 & 3940 & 97977 & 4.02 \\
\hline \multirow[t]{6}{*}{$1996 / 97$} & Overall & 29657 & 2915701 & 1.02 & 7665 & 2439681 & 0.31 & 21992 & 476020 & 4.62 \\
\hline & 1 (lowest) & 6923 & 536994 & 1.29 & 1953 & 445984 & 0.44 & 4970 & 91010 & 5.46 \\
\hline & 2 & 6292 & 570020 & 1.10 & 1588 & 471174 & 0.34 & 4704 & 98846 & 4.76 \\
\hline & 3 & 5900 & 585817 & 1.01 & 1438 & 489536 & 0.29 & 4462 & 96281 & 4.63 \\
\hline & 4 & 5247 & 604976 & 0.87 & 1373 & 514647 & 0.27 & 3874 & 90329 & 4.29 \\
\hline & 5 (highest) & 5295 & 617894 & 0.86 & 1313 & 518340 & 0.25 & 3982 & 99554 & 4.00 \\
\hline \multirow[t]{6}{*}{$1997 / 98$} & Overall & 29093 & 2962864 & 0.98 & 7353 & 2479880 & 0.30 & 21740 & 482984 & 4.50 \\
\hline & 1 (lowest) & 6492 & 543138 & 1.20 & 1718 & 450861 & 0.38 & 4774 & 92277 & 5.17 \\
\hline & 2 & 6146 & 578039 & 1.06 & 1553 & 477838 & 0.33 & 4593 & 100201 & 4.58 \\
\hline & 3 & 5992 & 595680 & 1.01 & 1494 & 497929 & 0.30 & 4498 & 97751 & 4.60 \\
\hline & 4 & 5268 & 615855 & 0.86 & 1346 & 524320 & 0.26 & 3922 & 91535 & 4.28 \\
\hline & 5 (highest) & 5195 & 630152 & 0.82 & 1242 & 528932 & 0.23 & 3953 & 101220 & 3.91 \\
\hline \multirow[t]{6}{*}{$1998 / 99$} & Overall & 28734 & 3004793 & 0.96 & 7005 & 2515345 & 0.28 & 21729 & 489448 & 4.44 \\
\hline & 1 (lowest) & 6374 & 547319 & 1.16 & 1705 & 453859 & 0.38 & 4669 & 93460 & 5.00 \\
\hline & 2 & 6228 & 584853 & 1.06 & 1513 & 483276 & 0.31 & 4715 & 101577 & 4.64 \\
\hline & 3 & 5834 & 604478 & 0.97 & 1339 & 505456 & 0.26 & 4495 & 99022 & 4.54 \\
\hline & 4 & 5220 & 626032 & 0.83 & 1269 & 533292 & 0.24 & 3951 & 92740 & 4.26 \\
\hline & 5 (highest) & 5078 & 642111 & 0.79 & 1179 & 539462 & 0.22 & 3899 & 102649 & 3.80 \\
\hline \multirow[t]{6}{*}{$1999 / 00$} & Overall & 29031 & 3045708 & 0.95 & 7046 & 2551920 & 0.28 & 21985 & 493788 & 4.45 \\
\hline & 1 (lowest) & 7029 & 565883 & 1.24 & 1834 & 472421 & 0.39 & 5195 & 93462 & 5.56 \\
\hline & 2 & 6438 & 599296 & 1.07 & 1533 & 495686 & 0.31 & 4905 & 103610 & 4.73 \\
\hline & 3 & 5637 & 615998 & 0.92 & 1337 & 516961 & 0.26 & 4300 & 99037 & 4.34 \\
\hline & 4 & 4927 & 626252 & 0.79 & 1245 & 532007 & 0.23 & 3682 & 94245 & 3.91 \\
\hline & 5 (highest) & 5000 & 638279 & 0.78 & 1097 & 534845 & 0.21 & 3903 & 103434 & 3.77 \\
\hline
\end{tabular}


Table 6: Crude mortality data from 1994/95 to 2005/06 among men without diabetes aged 30 years or more, by neighbourhood income and age group (part 2 of 2 )

\begin{tabular}{|c|c|c|c|c|c|c|c|c|c|c|}
\hline \multirow[b]{2}{*}{ Year } & \multirow[b]{2}{*}{$\begin{array}{l}\text { Income } \\
\text { quintile }\end{array}$} & \multicolumn{3}{|c|}{ All ages ( $\geq 30 \mathrm{yr}$ ) } & \multicolumn{3}{|c|}{ Age 30-64 yr } & \multicolumn{3}{|c|}{ Age $\geq 65 \mathrm{yr}$} \\
\hline & & Deaths & Population & $\begin{array}{c}\text { Mortality, } \\
\%\end{array}$ & Deaths & Population & $\begin{array}{c}\text { Mortality, } \\
\%\end{array}$ & Deaths & Population & $\begin{array}{c}\text { Mortality, } \\
\%\end{array}$ \\
\hline \multirow[t]{6}{*}{$2000 / 01$} & Overall & 28524 & 3093778 & 0.92 & 7098 & 2595968 & 0.27 & 21426 & 497810 & 4.30 \\
\hline & 1 (lowest) & 6549 & 571209 & 1.15 & 1736 & 477321 & 0.36 & 4813 & 93888 & 5.13 \\
\hline & 2 & 6243 & 607324 & 1.03 & 1557 & 502878 & 0.31 & 4686 & 104446 & 4.49 \\
\hline & 3 & 5890 & 625668 & 0.94 & 1409 & 525763 & 0.27 & 4481 & 99905 & 4.49 \\
\hline & 4 & 4988 & 638141 & 0.78 & 1253 & 543137 & 0.23 & 3735 & 95004 & 3.93 \\
\hline & 5 (highest) & 4854 & 651436 & 0.75 & 1143 & 546869 & 0.21 & 3711 & 104567 & 3.55 \\
\hline \multirow[t]{6}{*}{$2001 / 02$} & Overall & 27964 & 3147268 & 0.89 & 7044 & 2645379 & 0.27 & 20920 & 501889 & 4.17 \\
\hline & 1 (lowest) & 6470 & 578357 & 1.12 & 1715 & 484161 & 0.35 & 4755 & 94196 & 5.05 \\
\hline & 2 & 6281 & 616450 & 1.02 & 1594 & 511325 & 0.31 & 4687 & 105125 & 4.46 \\
\hline & 3 & 5510 & 636649 & 0.87 & 1309 & 535630 & 0.24 & 4201 & 101019 & 4.16 \\
\hline & 4 & 4755 & 650482 & 0.73 & 1217 & 554743 & 0.22 & 3538 & 95739 & 3.70 \\
\hline & 5 (highest) & 4948 & 665330 & 0.74 & 1209 & 559520 & 0.22 & 3739 & 105810 & 3.53 \\
\hline \multirow[t]{6}{*}{$2002 / 03$} & Overall & 27817 & 3199429 & 0.87 & 7200 & 2692569 & 0.27 & 20617 & 506860 & 4.07 \\
\hline & 1 (lowest) & 6426 & 583866 & 1.10 & 1757 & 488975 & 0.36 & 4669 & 94891 & 4.92 \\
\hline & 2 & 6151 & 625123 & 0.98 & 1540 & 518891 & 0.30 & 4611 & 106232 & 4.34 \\
\hline & 3 & 5528 & 647213 & 0.85 & 1363 & 545410 & 0.25 & 4165 & 101803 & 4.09 \\
\hline & 4 & 4883 & 663652 & 0.74 & 1318 & 566849 & 0.23 & 3565 & 96803 & 3.68 \\
\hline & 5 (highest) & 4829 & 679575 & 0.71 & 1222 & 572444 & 0.21 & 3607 & 107131 & 3.37 \\
\hline \multirow[t]{6}{*}{$2003 / 04$} & Overall & 28625 & 3240315 & 0.88 & 7420 & 2728376 & 0.27 & 21205 & 511939 & 4.14 \\
\hline & 1 (lowest) & 6443 & 586898 & 1.10 & 1843 & 491175 & 0.38 & 4600 & 95723 & 4.81 \\
\hline & 2 & 6269 & 631816 & 0.99 & 1607 & 524429 & 0.31 & 4662 & 107387 & 4.34 \\
\hline & 3 & 5708 & 655424 & 0.87 & 1405 & 552685 & 0.25 & 4303 & 102739 & 4.19 \\
\hline & 4 & 4985 & 674460 & 0.74 & 1294 & 576907 & 0.22 & 3691 & 97553 & 3.78 \\
\hline & 5 (highest) & 5220 & 691717 & 0.75 & 1271 & 583180 & 0.22 & 3949 & 108537 & 3.64 \\
\hline \multirow[t]{6}{*}{$2004 / 05$} & Overall & 27831 & 3278706 & 0.85 & 7273 & 2761626 & 0.26 & 20558 & 517080 & 3.98 \\
\hline & 1 (lowest) & 6398 & 601671 & 1.06 & 1796 & 508979 & 0.35 & 4602 & 92692 & 4.96 \\
\hline & 2 & 5946 & 647698 & 0.92 & 1610 & 541992 & 0.30 & 4336 & 105706 & 4.10 \\
\hline & 3 & 5425 & 657080 & 0.83 & 1421 & 555739 & 0.26 & 4004 & 101341 & 3.95 \\
\hline & 4 & 5172 & 682554 & 0.76 & 1277 & 578830 & 0.22 & 3895 & 103724 & 3.76 \\
\hline & 5 (highest) & 4890 & 689703 & 0.71 & 1169 & 576086 & 0.20 & 3721 & 113617 & 3.28 \\
\hline \multirow[t]{6}{*}{$2005 / 06$} & Overall & 28140 & 3306802 & 0.85 & 7291 & 2789696 & 0.26 & 20849 & 517106 & 4.03 \\
\hline & 1 (lowest) & 6561 & 603009 & 1.09 & 1945 & 510736 & 0.38 & 4616 & 92273 & 5.00 \\
\hline & 2 & 6052 & 651430 & 0.93 & 1560 & 545865 & 0.29 & 4492 & 105565 & 4.26 \\
\hline & 3 & 5233 & 662889 & 0.79 & 1309 & 561675 & 0.23 & 3924 & 101214 & 3.88 \\
\hline & 4 & 5243 & 690377 & 0.76 & 1282 & 586364 & 0.22 & 3961 & 104013 & 3.81 \\
\hline & 5 (highest) & 5051 & 699097 & 0.72 & 1195 & 585056 & 0.20 & 3856 & 114041 & 3.38 \\
\hline
\end{tabular}

